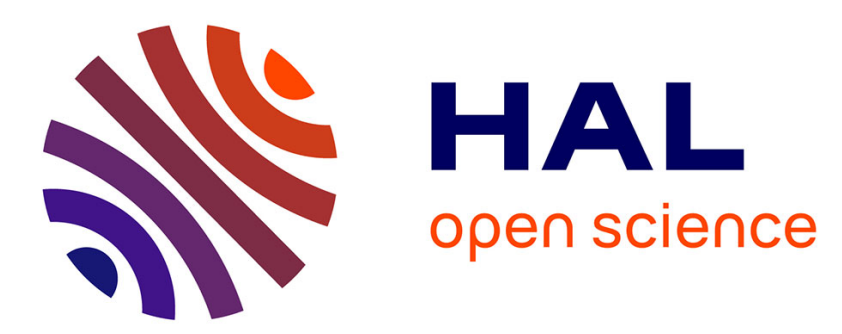

\title{
Adhesive Sponge Based on Supramolecular Dimer Interactions as Scaffolds for Neural Stem Cells
}

\author{
Luanda Lins, Florence Wianny, Colette Dehay, Jacques Jestin, Watson Loh
}

\section{To cite this version:}

Luanda Lins, Florence Wianny, Colette Dehay, Jacques Jestin, Watson Loh. Adhesive Sponge Based on Supramolecular Dimer Interactions as Scaffolds for Neural Stem Cells. Biomacromolecules, 2020, 21 (8), pp.3394-3410. 10.1021/acs.biomac.0c00825 . hal-03026544

\section{HAL Id: hal-03026544 https://hal.science/hal-03026544}

Submitted on 14 Dec 2020

HAL is a multi-disciplinary open access archive for the deposit and dissemination of scientific research documents, whether they are published or not. The documents may come from teaching and research institutions in France or abroad, or from public or private research centers.
L'archive ouverte pluridisciplinaire HAL, est destinée au dépôt et à la diffusion de documents scientifiques de niveau recherche, publiés ou non, émanant des établissements d'enseignement et de recherche français ou étrangers, des laboratoires publics ou privés. 


\title{
Adhesive Sponge Based on Supramolecular Dimers Interactions as Scaffolds for
}

\section{$\underline{\text { Neural Stem Cells }}$}

Luanda LINS ${ }^{a}$, Florence WIANNY' ${ }^{b}$, Colette DEHAY Jacques Jestin ${ }^{c}$, Watson Loh ${ }^{a}$.

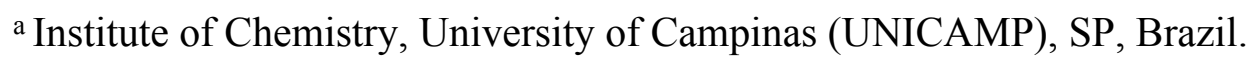

b Univ Lyon, Université Claude Bernard Lyon 1, INSERM, Stem Cell and Brain

Research Institute U1208, 69500 Bron, France.

` Laboratoire Léon Brillouin, UMR12, Bat 563 CEA Saclay, 91191 Gif sur Yvette Cedex, France.

*Corresponding to: Luanda Lins.E-mail address: luandaqmc@gmail.com

Keywords: Supramolecular, 2-ureido-4-pyrimidone, Sponge-like scaffolds, Neural Tissue Engineering.

\begin{abstract}
$\underline{\text { Abstract }}$
Improving cell-material interactions of non-adhesive scaffolds is crucial for the success of biomaterials in tissue engineering. Due to their high surface area and open pore structure, sponges are widely reported as absorbent materials for biomedical engineering. The biocompatibility and biodegradability of polysaccharides sponges, coupled with the chemical functionalities of supramolecular dimers, make them promising combinations for the development of adhesive scaffolds. Here, a supramolecular tactic based on (UPy)-modified polysaccharide associated with threedimensional structure of sponges was developed to reach enhanced cellular adhesion. For this purpose, three approaches were examined individually in order to accomplish this goal. In the first approach, the backbone polysaccharides with non-cell adhesive properties were modified via a modular tactic using UPy-dimers. Hereupon, the
\end{abstract}


physical-chemical characterizations of the supramolecular sponges were performed, showing that the presence of supramolecular dimers improved their mechanical properties and induced different architectures. In addition, small-angle neutron scattering (SANS) measurements and rheology experiments revealed that the UPydimers into agarose backbone are able to reorganize in thinning aggregates. It is also demonstrated that the resulted UPy-agarose (AGA-UPy) motifs in surfaces can promote cell adhesion. Finally, the last approach showed the great potential for use of this novel material in bioadhesive scaffolds indicating that neural stem cells show a spreading bias in soft materials and that cell adhesion was enhanced for all UPy-modified sponges compared to the reference, i.e. unmodified sponges. Thus, by functionalizing sponge surfaces with UPy-dimers, an adhesive supramolecular scaffold is developed which opens the opportunity its use improving neural tissues regeneration.

\section{Introduction}

Neural tissue engineering (NTE) is an emerging area at the interfaces of chemistry, engineering materials, neuroscience, and biology, where research tools in cell biology are combined with engineering biomaterials principles to facilitate the cell adhesion on scaffold for regeneration or repair of neural tissues. The combination of neural stem cells, biomaterial scaffolds, and bioadhesive molecules may contribute to cultivate cells in vitro before being implanted into damaged tissue. The scaffold implanted can stimulate the self-healing capability of the tissue for neural system repair in different ways. Besides, monkey Neural Stem Cells (NSCs) on a suitable scaffold may be implanted on the brain with a lessened risk of immune rejection, representing invaluable implements for preclinical studies involving neural tissue engineering 1, 2 . There seems to be a general consensus that a three-dimensional scaffold with a suitable 
microstructure is essential to promote cell adhesion, proliferation, and driving tissue regeneration as well as to support oxygen exchange and nutrient diffusion. Additionally, scaffolds need to exhibit rough surfaces and high porosity to promote chemical bonding with the tissue associated with the proper mechanical features to support the cells and to meet the required achievement of the tissues at the site of implantation. Thanks to their large surface area, high absorption capacity, and high porosity, sponges or aerogels are good candidates as scaffolds for these cells growth ${ }^{3,4}$.

Superabsorbent and porous scaffolds, such as sponges or aerogels, can be yielded at frozen temperatures from polymeric hydrogels, of which the hydrophilic structure provides them adept of holding huge amounts of water in their threedimensional structures. So far, polysaccharide hydrogels have been explored as biomaterials for three-dimensional cell encapsulation and transplantation. This is due to fact that these hydrogels can resist to compressive forces on the matrix while permitting a fast diffusion of nutrients between the blood and tissue cells. Agarose is a typical example of hydrogel that forms a non-cytotoxic support. It may be utilized to reproduce the natural cells environment, as extracellular matrix (ECM). However, agarose and other hydrophilic polysaccharides form a stiff inert hydrogel that does not carry any biological information, thus the cells cannot adhere to the surface of these scaffolds ${ }^{5}$. Therefore, great challenges still remain to transform hydrophilic polymer-based hydrogels to equivalent bioadhesive sponges.

One strategy to enhance the integration of cells and tissues into scaffolds, involves the use of adhesion molecules by functionalization of the scaffold surfaces. Biomaterials may be adapted with small recognition motifs that mimic the ECM to endorse cell binding. Therein, incorporating adhesion molecules into polymers backbone is an excellent methodology for growing tissue integration of neural 
biomaterials ${ }^{6}$. It has been already reported that grafting short motifs to hydrogels improves neural cell adhesion, while conserving a matrix with similar properties to those found in vivo ${ }^{7-9}$. In this present work, supramolecular dimer 2-ureido-4(1H)pyrimidone (UPy) was used as a source of active functional groups into agarosederivative chains, resulting in an improvement in the scaffold physical properties and greater ability in neural cell adhesion on surfaces. UPy-dimer is a self-complementary quadruple hydrogen bonding motif based on donor-acceptor array, obtaining a molecule with four hydrogen-bonding sites and providing homo-complementary sequences ${ }^{10}$. Recent articles have shown that supramolecular materials based on UPy-dimers quadrupole-hydrogen bonds are signally appropriate for yielding such bioactive scaffolds owing to their low-temperature processability, adhesive capacity, biocompatible behavior, and favorable degradation 11-15. Particularly, the functionalization of scaffolds surfaces with UPy-dimers can promote rapid communication with integrins through physical adsorption by intermediate proteins and other biological molecules favoring cells signal transduction ${ }^{15}$. Thereby, the dynamic nature of the supramolecular hydrogen bonds agrees with a modular tactic to gaining control over cellular performance and activity both in vitro and in vivo.

The interactions via supramolecular hydrogen bonds have attracted attention in science biomaterials due their enormous impact on our daily life. The pairing of nucleic acid strands in DNA is possibly the most sophisticated example of supramolecular interactions in nature, as it is based in cooperative multiple hydrogen bonding between bases and hydrophobic interactions. Likewise UPy, the combination of auto-associative systems, as $-\mathrm{NH}_{2},-\mathrm{O},-\mathrm{H}$, and $-\mathrm{N}$ form dynamic dimers. Faced with this phenomenon, UPy-dimers can also be used as a new alternative 'crosslinker' for polysaccharides chains to adjust the microstructure of the sponge. The occurrence of dimers junction 
zones from the hydrogen bonds and hydrophobic interactions between polymer chains renders the material softer and less brittle. The simplest methodology to creating a surface-based supramolecular structure in sponges is to use UPy-dimers in agarose backbone capable of creating an extended hydrogen bond network without the need of adding any additional agents. The hypothesis is that the mildly different chemical structures of the agarose linked to UPy will result in a substantial difference in the sponge microarchitecture providing the same rapid gelation; this difference will then alter the behavior of neural stem cells seeded in the scaffolds. We report here a structurally new type of sponge-like scaffold, a supramolecular sponge, derived from homo-complimentary hydrogen bonding. This is a modular method based on the supramolecular dimers to form sponges-like scaffolds with tailorable properties to help adhesion and cell growth in scaffolds.

\section{Experimental section}

\subsection{Materials}

Agarose for molecular biology (low EEO), 2-Amino-4-hydroxy-6methylpyrimidine, 1,6-Diisocyanatohexane $98 \%$, Pentane, Pyridine, N,NDimethylformamide - anhydrous, $99.8 \%$ were purchased SIGMA-Aldrich.

\subsection{Synthesis}

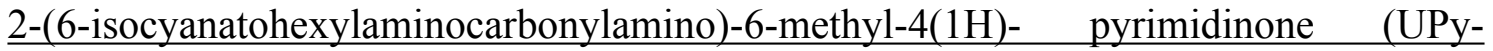
NCO):

The synthesis of 2-(6-isocyanatohexylaminocarbonylamino)-6-methyl-4 (1H) pyrimidinone (UPy-NCO) was adapted from Meijer et al ${ }^{16}$. A mixture of 2-amino-4- 
hydroxy-6-methylpyrimidine (5 g, $0.03995 \mathrm{~mol})$ in excess of 1,6-hexanediisocyanate (50 g, $0.2972 \mathrm{mols})$ and pyridine $(3 \mathrm{~g}, 3.06 \mathrm{~mL})$ was agitated during 16 hours at $100{ }^{\circ} \mathrm{C}$ under argon atmosphere. A white solid was obtained after pyridine removal and excess diisocyanate by filtration in a sintered-glass filter and then a succession of washes with pentane $(500 \mathrm{~mL})$. The product obtained was then dried at $50^{\circ} \mathrm{C}$ to remove any trace of water (in a Schlenk tube under argon). A yield of $95.5 \%$ was determined.

General procedure for the synthesis of Agarose-UPy

The general synthesis of agarose backbone functionalization with UPy (AGA$\mathrm{Br}, \mathrm{AGA}-\mathrm{N}_{3}, \mathrm{AGA}-\mathrm{NH}_{2}$, and AGA-UPy) is detailed in the in the Supporting Information.

\subsection{Preparation of sponge-like scaffolds with alternating degrees of supramolecular cross-linking}

For the preparation of supramolecular crosslinked gels, $0.25 \mathrm{~g}$ of different ratios between neat agarose and synthetized AGA-UPy were dispersed in $5.0 \mathrm{~mL}$ deionized water and stirred at $90{ }^{\circ} \mathrm{C}$ until a fully dispersion was obtained. To obtain a lower and higher degree of supramolecular cross-linking, the 90:10 and 50:50 ratios of agarose and synthetized AGA-UPy (AGA:AGA-UPy) were chosen for this study. For better analysis of the results, neat agarose hydrogel was also prepared and characterized. The gelation of the samples was confirmed by the standard inversion test. To prepare sponges, the mixtures with AGA-UPy and neat agarose were poured into PP molds at $20{ }^{\circ} \mathrm{C}$ during $24 \mathrm{~h}$, and subsequently freeze-dried to remove ice by sublimation.

\subsection{Characterizations}


The FTIR of 6-methyl-isocytosine and 2-(6-isocyanatohexylaminocarbonylamino)6-methyl-4(1H)- pyrimidinone were finely ground and then mixed with potassium $(\mathrm{KBr})$ in order to form pellets by vacuum pressure. The spectra were collected at a resolution of 32 scans and $4 \mathrm{~cm}^{-1}$ with Nicolet iS10 thermo scientific spectrometer in transmission mode from $4000-650 \mathrm{~cm}^{-1}$.

${ }^{1} \mathbf{H}$ spectra was performed on a Bruker Avance III $400 \mathrm{MHz}, 500 \mathrm{MHz}$ or AvanceNEO $600 \mathrm{MHz}$ spectrometer. Samples were dissolved in deuterated DMSO. The coupling constants are indicated in $\mathrm{Hz}$ and chemical shifts $(\delta)$ are expressed in ppm, relative to internal tetramethylsilane for $1 \mathrm{H}$ nuclei. The solid-state ${ }^{\mathbf{1 3}} \mathbf{C}$ NMR spectra was recorded at $11.7 \mathrm{~T}$ magnetic field using an AVANCE III HD spectrometer at Larmor frequencie $v(13 \mathrm{C})=125.783 \mathrm{MHz}$ using a $4 \mathrm{~mm}$ cross-polarization magic angle spinning probe (CP-MAS). MAS spinning speeds for all samples were $10 \mathrm{kHz}$.

The reaction between UPy-NCO and Agarose was evaluated using a K-Alpha+ XPS spectrometer (ThermoFisher Scientific, UK) with monochromatic Al Ka X-ray radiation with pass energy of $200 \mathrm{eV}$ for survey size (400 $\mu \mathrm{m}$ spot) and $50 \mathrm{eV}$ for highenergy resolution core level spectra. Spectra were recorded with an angle of incidence of $30^{\circ}$ at two locations and elemental compositions were determined from lowresolution survey measurements with $200 \mathrm{eV}$ for survey and $50 \mathrm{eV}$ for high-energy resolution core level spectra. The Scofield sensitivity factors, analyzer transmission function, and effective attenuation lengths (EALs) for photoelectrons were applied for quantification. EALs were calculated using the standard TPP-2M formalism, which were controlled by means of the well-known photoelectron peaks of metallic $\mathrm{Cu}, \mathrm{Ag}$, and $\mathrm{Au}$.

$$
\% N_{\text {sub }}=\frac{N_{\text {exp } \%}}{N_{\text {theorical }}} \times 100 \quad \text { (Equation 1) }
$$


Thermogravimetric Analyses (TGA) of UPy-NCO, AGA-NH ${ }_{2}$, AGA-UPy and the AGA-NH $2 /$ UPy-NCO physical mixture were recorded on a Q500 thermogravimetric analyzer (TA instruments). The samples were heated from 25 to $800{ }^{\circ} \mathrm{C}$ at a rate of 20 ${ }^{\circ} \mathrm{C} \min ^{-1}$ under $\mathrm{N}_{2}$ flow.

Differential Scanning Calorimetry measurements (DSC) of neat UPy-NCO, functionalized agarose i.e. AGA-UPy, AGA-NH$H_{2}$ and the AGA-NH2/UPy-NCO mixture were performed on a Q20 (TA instruments) from 10 to $140{ }^{\circ} \mathrm{C}$ to prevent any thermal degradation of the compounds as demonstrated by TGA. The samples were heated or cooled at a rate of $10 \mathrm{~K} \mathrm{~min}^{-1}$ under $\mathrm{N}_{2}$ flow of $50 \mathrm{~mL} \mathrm{~min}{ }^{-1}$.

Hydrogels rheological analyses were carried out using a Rheometric Scientific ARES instrumentation at $37{ }^{\circ} \mathrm{C}$ equipped with $30 \mathrm{~mm}$ parallel plates, with a $4 \mathrm{~mm}$ gap between them (TA Instruments, New Castle, DE, US). The oscillatory responses $\left(\mathrm{G}^{\prime}=\right.$ elastic modulus and $\mathrm{G}^{\prime \prime}=$ loss/viscous modulus) were determined at low values of strain $(0.02 \%)$ over the frequency range $0.1-100 \mathrm{rad} / \mathrm{s}$.

The compression strength of the cylindric sponges were measured in a compression testing machine (thermomechanical analyzer TMA 2940 TA Instruments). The tests were performed at a compression at a constant rate of $0.05 \mathrm{~N} \mathrm{~min}^{-1}(20 \mathrm{~mm}$ DIA cylinder aluminium), equilibrium at $25^{\circ} \mathrm{C}$.

The high resolution X-ray micro-computed tomography (Resolution: 1 pixel $=4.5$ um) (uCT; model Skyscan 1272, Bruker microCT, Kontich, Belgium) was used to reconstruct three-dimensionally the internal structure of sponges like-scaffold.

The Small-angle neutron scattering (SANS ) measurements were performed on the small-angle spectrometer PA20 spectrometer of Leon Brillouin Laboratory (LLB, Saclay, France). The three configurations are defined by a constant wavelength $\lambda=6 \AA$, three sample-to-detector distances $(2,8$, and $19 \mathrm{~m})$. These setups enable to cover a total 
q-range from $2.10^{-3}$ to $0.3 \AA^{-1}$. Hydrogels were placed into standart Hellma cells in a sample changer and measured under ambient conditions. The 2D patterns were reduced to $1 \mathrm{D}$ spectra, I(q) versus $\mathrm{q}$, after a radial averaging around the center of the scattered beam. Neutron beam transmission, standard corrections by sample thickness, electronic background, empty beam signal subtraction, detector efficiency, and subtraction of incoherent scattering were applied to get the scattered intensities on an absolute scale $\left(\mathrm{cm}^{-1}\right)$.

To measure the water retention capacity, the sponges were placed in water at $37^{\circ} \mathrm{C}$ overnight, then the excessive surface water was removed by gently tapping the sponges on a paper towel and $W_{\text {wet }}$ was recorded. Sponges were dried at $60{ }^{\circ} \mathrm{C}$ overnight, and the $W_{d r y}$ was measured. The succeeding equation was used to calculate the percent water holding of the sponges:

$$
W R(\%)=\frac{W_{w e t}-W_{d r y}}{W_{d r y}} \times 100
$$

(Equation 2)

Scanning Electron Microscopy (SEM Philips XL30) was used to detect the surface changes in sponges induced by UPy-dimers addition.

\subsection{Neural stem cell culture}

\section{Neural Stem Cells:}

Neural Stem Cells (NSCs) were previously isolated in our laboratory from monkey embryonic stem cells (ESCs) ${ }^{18}$. Concisely, monkey ESCs were cultured to confluency during 15 days with daily medium change. Neuroepithelial-like cells spontaneously emerging in culture were designated manually and transferred into gelatin coated dishes (0.1\%; Sigma), and cultured in "NSC medium" (N2 supplement, DMEM/F12, $1 \%$ 
nonessential amino acids, $2 \mathrm{mM}$ L-glutamine, $0.1 \mathrm{mM} \beta$-mercaptoethanol (Invitrogen), with $20 \mathrm{ng} / \mathrm{ml} \mathrm{FGF2} \mathrm{(Millipore)} \mathrm{and} 20 \mathrm{ng} / \mathrm{ml}$ EGF (Millipore). NSCs used in this study stably express the TAU-green fluorescent fusion protein (TAU-GFP), which binds the GFP to microtubules. For amplification, NSCs were trypsinized (trypsin 0,025\%, EDTA, 0,1 g/L) (Invitrogen). Trypsin was inhibited by addition of Trypsin Inhibitor (Invitrogen) and cells were resuspended in NSC medium.

Cell loading:

Sponge-like scaffolds were UV treated for 30 minutes, and 2-mm thick sections were placed into 24-well dishes. NSCs were harvested and resuspended at a density of 2 $10^{6} \mathrm{NSCs} / \mathrm{ml}$ in NSC medium. An aliquot of $50 \mu \mathrm{L}$ was added on top of each spongelike sample. After 2 mins incubation, another $50 \mu \mathrm{L}$ aliquot was added $\left(2.10^{5} \mathrm{NSCs}\right.$ total). Sponge-like scaffolds were let to swell for 5 mins in the incubator at $37^{\circ} \mathrm{C}$ and $900 \mu \mathrm{l}$ of NSC medium was added. The loaded samples were incubated at $37^{\circ} \mathrm{C}, 5 \%$ $\mathrm{CO}_{2}, 5 \% \mathrm{O}_{2}$, for 3 days, replacing medium every other day.

\section{$\underline{\text { Immunofluorescent stainings }}$}

Sponge-like scaffolds were fixed by immersion in $2 \%$ paraformaldehyde in cold phosphate-buffer at $4{ }^{\circ} \mathrm{C}$ for $30 \mathrm{~min}$, and embedded in $3 \%$ low gelling agarose (Sigma) in HBSS (Life Technologies) supplemented with glucose $18 \%, \mathrm{MgSO}_{4}$ and $\mathrm{CaCl}_{2}$ (Sigma) at $37{ }^{\circ} \mathrm{C} .100 \mu \mathrm{m}$ thick slices were prepared with a vibratome (Leica VT1000S).

Slices were permeabilized with Triton X-100 (0.1\% in Tris-buffered saline (TBS). Aspecific binding was blocked by incubation in normal donkey serum (10\% in TBS) (Jackson Immunoresearch Laboratories, West Grove, PA, USA) for $20 \mathrm{~min}$ at room temperature (RT). Slices were incubated overnight at $4{ }^{\circ} \mathrm{C}$ with anti-GFP $(1 / 800$, 
A10262, Life Technologies) diluted in antibody diluent (Dako, Glostrup, Denmark). After three rinses in TBS, they were incubated with affinity-purified donkey antiChicken conjugated to Alexa488 (Life Technologies) for $1 \mathrm{~h}$ at RT. To stain F-actin, slices were incubated with Phalloidin-Alexa Fluor 488 (Life Technologies) diluted in antibody diluent, for 45 mins at RT. Nuclei were counterstained with 4', 6-diamidino-2phenylindole (DAPI) (D103; 1/5000; Life Technologies) for 3 mins. Slices were mounted on slides in Fluoromount G (Clinisciences). Images were acquired by confocal microscopy (Leica, TCS SP at 10X, 20X, and 40X objectives). Z-stack images were acquired at 5 to $10 \mu \mathrm{m}$ intervals.

\section{Results and Discussion}

\subsection{Synthesis and characterization of the associative unit and precursor comonomer of a supramolecular foams network}

Synthesis and characterization of 2-(6-isocyanatohexylaminocarbonylamino)-6-methyl$\underline{4(1 H) \text { - pyrimidinone }(U P y-N C O)}$

Ureidopyrimidinone dimers (UPy) are formed by strong quadruple hydrogen bonds capable of physically crosslinking a material network and at the same time, these physical links can be reversibly broken and reformed. This principle was chosen by Meijer et al. who have synthesized isocytosine derivatives able of associating in the same way as the pyrimidines and purine sequences, i.e. donor-acceptor homocomplementary interaction (DDAA) ${ }^{19}$. Isocytosine has an amine function, whose reaction with an isocyanate forms a new hydrogen bond by the creation of a urea function. This gives the site four hydrogen bonds, whose spatial arrangement is favored by the presence of an intramolecular hydrogen bond. The UPy donor-acceptor 
interaction (DA) between neutral electron-deficient and electron-rich dimers can be fruitfully used to the self-assembly of various interconnected supramolecular materials.

The product which we will be named in this paper as UPy-NCO is derived from the condensation reaction of isocytosine in hexane diisocyanate (as shown in Figure S1) 20, 21. The excess of diisocyanate (dilution effect) combined with a substitution effect (decreased reactivity of the second isocyanate function) promoted the formation of monofunctional species. On the basis of ${ }^{1} \mathrm{H}$ NMR analysis (Figure S1), the UPy moieties composition was confirmed showing a good agreement with the proposed structure. The attendance of peaks at $\delta=10.18,11.85$, and $13.01 \mathrm{ppm}$ which were used to ascribe the $\mathrm{NH}$ proton signals, confirming the $4[1 \mathrm{H}]-$ pyrimidinone dimer geometry in solution ${ }^{19}$.

The product functional groups have characteristic absorbance peaks at perfectly identifiable wavelengths as shown in Figure 1. After synthesis, the $\mathrm{NH}_{2}$ peak at $\sim 3000$ $\mathrm{cm}^{-1}$ disappeared in the spectrum of UPy-NCO, and new peaks emerged at $\sim 3400-3200$ $\mathrm{cm}-1$, are assigned to the NH vibrations (amide band I, and II) ${ }^{16,19}$. Noteworthy, a band at $2283 \mathrm{~cm}^{-1}$ reveals the presence of the isocyanate function ${ }^{22}$. The analysis of the carbonyl group region in UPy-NCO reveals the presence of two $1700 \mathrm{~cm}^{-1}$ (amide I) absorption bands originating from the formation of the urea function and to the carbonyl isocytosine at1666 $\mathrm{cm}^{-1}$. Finally, the presence of the two 1600 and $1522 \mathrm{~cm}^{-1}$ bands characteristic of the amide II vibration modes indicates the presence of a N, N' bisubstituted urea function ${ }^{22}$. 


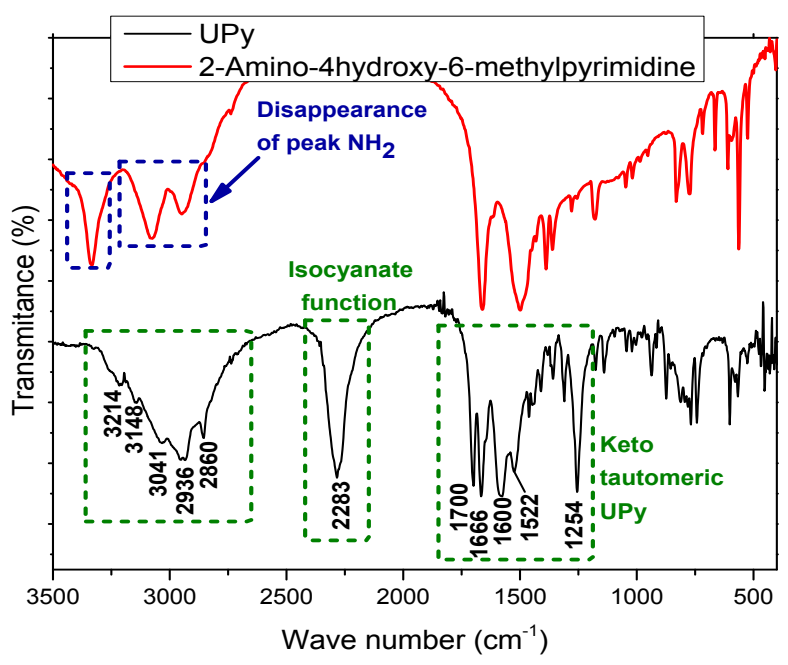

Figure 1. Infrared spectra of 2-(6-isocyanatohexylaminocarbonylamino)-6methyl-4(1H)- pyrimidinone.

\section{Supramolecular Agarose Functionalization: Agarose-UPy}

Agarose polysaccharide was selected as a cryogel base for scaffolds building due to its ability to immobilize water molecules and its inertness. Polysaccharides with functionalized groups, e.g., $\mathrm{SH}, \mathrm{NH}_{2}$ and $\mathrm{COOH}$, have many medical, biological, and biotechnological applications. Amino-polysaccharides can be used as synthetic tools owing to their structural specificity to isolate carbohydrate-binding proteins and biomolecules ${ }^{23}$. The high regio-selective introduction of the amino group in agarose and their derivatives is extremely important to coupling reactions. Here, we present a facile synthetic approach using 'click chemistry' to produce functional 6-amino-agarose $\left(\mathrm{AGA}-\mathrm{NH}_{2}\right)$ via 6-bromo-deoxyagarose $(\mathrm{AGA}-\mathrm{Br})$. In this synthetic regio-selective approach, unmodified agarose was first brominated with carbon tetrabromide and triphenylphosphine; and then azidated with sodium azide to afford 6-azido-6deoxyagarose $\left(\mathrm{AGA}-\mathrm{N}_{3}\right)$. The synthetic route for 6-amino-6-agarose from $(1,3)$ linked D- $\beta$-galactose $(1,4)$ linked- $\alpha$-anhydro galactose is shown in Figure S2. Agarose-amine functionalization and characterization are presented in more details at Support 
Information. The subsequent coupling reaction occurs between electrophilic isocyanate and common nucleophilic end groups of telechelic polymers as amine groups. The molecule denoted (UPy-NCO) readily reacts with amine functions (urea formation) to form the novel AGA-UPy, as shown in Scheme 1.

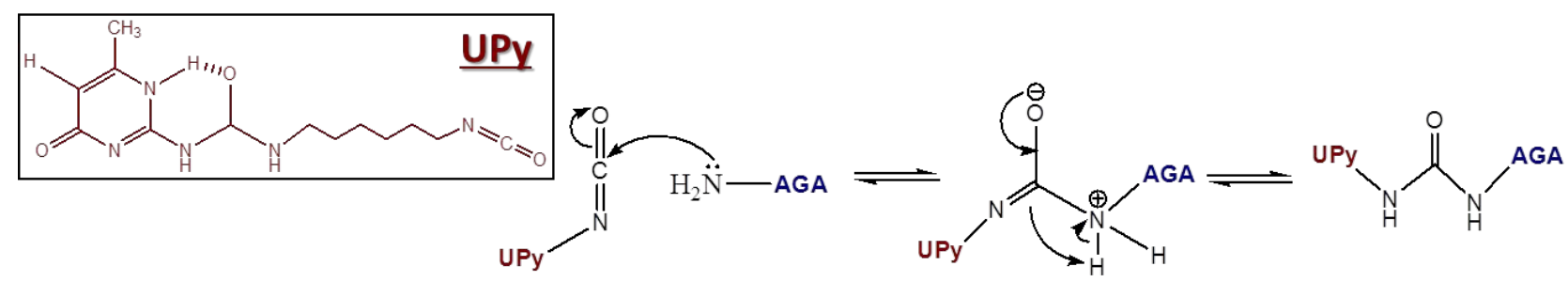

Schema 1. Synthesis of the Agarose-UPy via SN2 from the formation of urea.

FT-IR and solid-state 13C NMR were performed to confirm the chemical structure of unmodified agarose, AGA-NH $\mathrm{NH}_{2}$ (Figure $\mathrm{S} 3$ and table S1), and AGA-UPy. The FTIR spectra in Figure 2 shows total disappearance of the $2285 \mathrm{~cm}^{-1}$ characteristic isocyanate function $(\mathrm{N}=\mathrm{C}=\mathrm{O})$ band associated with the appearance of a new $1590 \mathrm{~cm}^{-1}$ peak characteristic of urea, clearly confirming the success of the functionalization ${ }^{24}$. These results indicate the formation of AGA-UPy without presence of UPy-NCO ${ }^{25}$. The new bands emerging in the spectral region of 3050 and $2850 \mathrm{~cm}^{-1}$ are characteristic of the secondary amines $(\mathrm{N}-\mathrm{H})$ of urea formation. These absorptions bands suggest that they are predominantly present in the form covalently associated with the agarose. In the spectral region from wavenumbers $1700-1500 \mathrm{~cm}^{-1}$, in the case of $\mathrm{AGA}-\mathrm{NH}_{2}$, only a single broad band of absorption can be observed. Instead, the functionalization by UPy leads to the appearance of many bands in this region. The presence of UPy characteristic bands $\left(\mathrm{n}=1700-1666-1590-1523 \mathrm{~cm}^{-1}\right)$ reveals the presence of the UPy associative entity. 


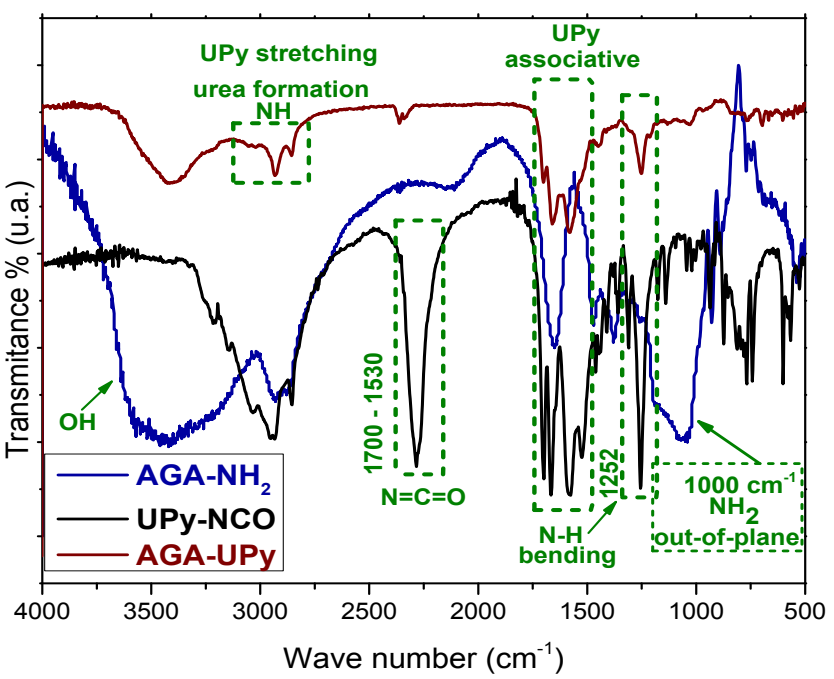

Figure 2. FTIR of amine-agarose (AHA-NH $\mathrm{NH}_{2}$, UPy-NCO precursor, and Agarose-UPy (AGA-UPy).

To further confirm the coupling reaction to form UPy-grafted agarose, the solid state ${ }^{13} \mathrm{C}$ NMR spectra of products were examined. As shown in Figure S4, the ${ }^{13} \mathrm{C}$ NMR solid-state spectra of the unmodified agarose as well as its peak assignments was confirmed by the literature ${ }^{26-28}$. The differences observed between unmodified agarose and AGA-UPy samples were perceptible in CP-MAS ${ }^{13} \mathrm{C}$ NMR spectra. For UPy signals, we can see peaks in the range of 50-30 ppm corresponding to the hydrocarbons present in the isocyanate structure; but we can mainly focus on the peak corresponding to the reactive isocyanate group present at $121 \mathrm{ppm}^{29,30}$. Isocyanates are very reactive compounds which react almost spontaneously with amino groups to form urea. In the AGA-UPy spectrum, the absence of the isocyanate peak is clear, indicating the subsequent coupling reaction between $\mathrm{AGA}-\mathrm{NH}_{2}$ and UPy-NCO, yielding the AGAUPy product. Certainly, this is caused by the urea linkage between agarose and UPymoieties, as shown in scheme 1.

$\mathrm{X}$-Ray photoelectron spectroscopy (XPS) is a highly sensitive tool which can provide data about the atomic composition and speciation of each atom on grafted 
agarose chains. Because XPS is a suitable technique for obtaining information of chemical structure of compounds in the solid state, the chemical environment of unmodified agarose, AGA-NH , and AGA-UPy could be analyzed without any interference from interactions in solution. In order to obtain a deeper insight, the high resolution of $\mathrm{C} 1 \mathrm{~s}$ and N1s spectra are shown in Figure 3 before and after agarose modification.

The deconvolution signals of unmodified agarose, agarose with $\mathrm{NH}_{2}$-grafted groups and sequentially coupled by UPy-moieties show that different covalent bonds can be associated with different carbon signal peaks. The survey spectra obtained of $\mathrm{C}(1 \mathrm{~s})$ from unmodified agarose illustrates binding energies at $285.0 \mathrm{eV}$ corresponding to alkane-type carbon $\mathrm{C}-\mathrm{H} / \mathrm{C}-\mathrm{C}, 286.4 \mathrm{eV}$ associated to $\mathrm{C}-\mathrm{O}$ of ether groups, and 288.0 $\mathrm{eV}$ which can be attributed to $\mathrm{C}-\mathrm{O}$ of alcohol groups ${ }^{31}$. AGA- $\mathrm{NH}_{2}$ spectra also exhibited a $\mathrm{C} 1 \mathrm{~s}$ binding energy peak at $285.0 \mathrm{eV} \mathrm{C}-\mathrm{O}$ carbon alkanes. Moreover, a shoulder peak at $284.6 \mathrm{eV}$ appears confirming covalent $\mathrm{C}-\mathrm{N}$ binding from the substitution of $\mathrm{OH}$ by $\mathrm{NH}_{2}$-groups on agarose-C6 ${ }^{32}$. After grafting $\mathrm{NH}_{2}$ groups into the polysaccharide backbone (C6), the peak corresponding to $\mathrm{C}-\mathrm{O}$ and $\mathrm{C}-\mathrm{N}$ became predominant, while the peak at 288.0 markedly decreased, indicating the modification of hydroxyl-containing functional groups ${ }^{33}$. $\mathrm{C} 1$ s component for remaining hydroxyl groups tends to be shifted to higher binding energy and a weak intensity relative to C-O and $\mathrm{C}-\mathrm{N}$, thus being able to be overlapped by these peaks. In AGA-UPy spectra, the successful UPy-functionalization on AGA- $\mathrm{NH}_{2}$ was also confirmed by similar binding energies for $\mathrm{C}-\mathrm{N}$ at $284.6 \mathrm{eV}$, whereas the peak at $288.4 \mathrm{eV}$ was assigned to carboxylic functions $(\mathrm{C}=\mathrm{O})$ originating from urea function borne by AGA-UPy binding.

The high-resolution XPS spectra of N1s (Figure 3) confirms amino-agarose functionalization and coupling reaction between $\mathrm{NH}_{2}$ and isocyanate groups of UPy- 
NCO moieties. Evidently, since unmodified agarose is composed solely of carbon and oxygen, no peak in N (1s) spectra are detectable. Instead, the spectra of modified agarose by $\mathrm{NH}_{2}$ and afterwards by UPy hihlights three peaks at $399.2\left(-\mathrm{NH}_{2}\right), 400.3(-$ $\mathrm{NH}$ ), and $401.7 \mathrm{eV}\left(\mathrm{NH}_{3}{ }^{+}\right)$, confirming the modification of agarose ${ }^{32}$. The synthesized AGA- $\mathrm{NH}_{2}$ are strongly dependent on the protonation state of the amine, and the XPS spectrum highlighted two different types of nitrogen corresponding to the presence of deprotonated primary amine $\left(-\mathrm{NH}_{2}, 399.5 \mathrm{eV}\right)$ as well as the protonated ammonium ($\left.\mathrm{NH}_{3}{ }^{+}, 401.5 \mathrm{eV}\right)$. These same results have been described in the literature for systems such as chitosan or glucosamine. For example, Wu et al. have highlighted by XPS measurements the formation of the deprotonated and protonated forms of the glucosamine at $399.5 \mathrm{eV}$ and $401.5 \mathrm{eV}$, respectively ${ }^{34,35}$. The estimated amine groups substitution using XPS can be calculated by Equation 1 (experimental section), and showed that $\mathrm{NH} 2$ functionalization after synthesis is estimated at $30 \%$. After the coupling reaction, the peak attributed to the $\mathrm{NH}_{2}$ is no longer observed and instead, two peaks appear. The peak at $400.1 \mathrm{eV}$ is attributed to secondary amide resulting from the coupling reaction (urea formation) between the isocyanate function of the UPy-NCO moieties and the primary amine at the agarose chain. Finally, N (s1) peak at $401.0 \mathrm{eV}$ corresponding to the primary amine of the UPy-moieties ${ }^{36}$. In conclusion, the modification of the agarose with amine groups was achieved and supramolecular AGAUPy was successfully synthesized. 

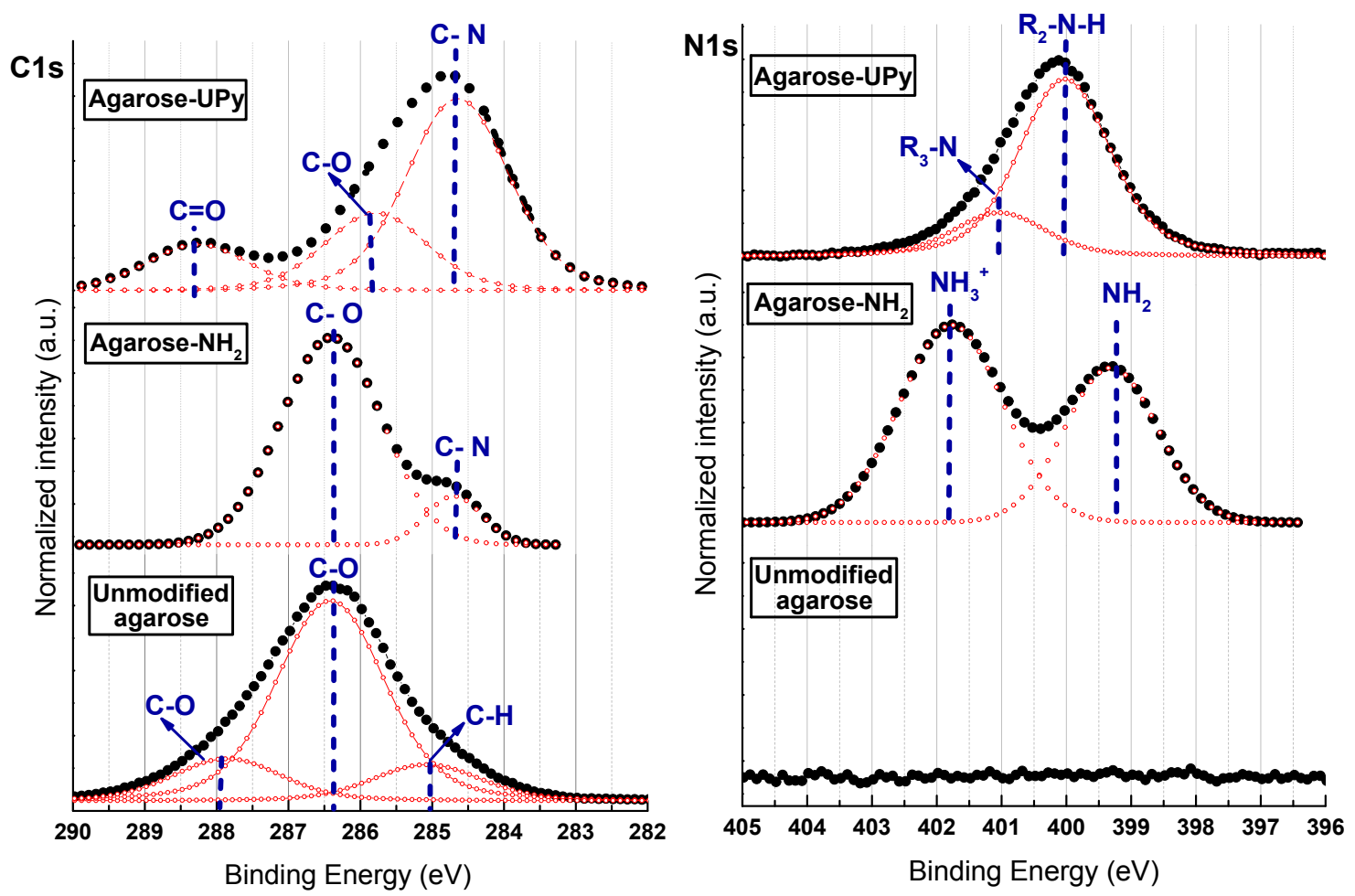

Figure 3. Deconvoluted high-resolution $\mathrm{C} 1 \mathrm{~s}$ and $\mathrm{N} 1 \mathrm{~s} \mathrm{X}$-ray photoelectron spectra of unmodified agarose, AGA-NH2 (amine-agarose), and AGA-UPy.

\subsection{Thermal Properties of AGA-UPy}

For a better AGA-UPy covalent linkage analysis, TGA was used to highlight the decomposition mechanisms of UPy-NCO and modified-agarose, i.e., AGA-NH 2 , AGAUPy, and AGA- $\mathrm{NH}_{2} / \mathrm{UPy}$ physical mixture. Thus, the TGA thermograms was presented in Figure 4 and the decomposition temperatures of all the systems are reported in Table S2. Three distinct degradation steps were observed for ureidopyrimidinone compound in Figure 4-A. The first degradation occurs between $170{ }^{\circ} \mathrm{C}$ and $268{ }^{\circ} \mathrm{C}$, representing 13 wt $\%$ and corresponds to the decomposition of the hexamethylene diisocyanate. Then, different degradation mechanisms take place between $210{ }^{\circ} \mathrm{C}$ and $400{ }^{\circ} \mathrm{C}$ corresponding to degradation of $59 \mathrm{wt} \%$ of UPy. In fact, it is well-known that the increase of 
temperature may result in the formation of isocyanurate functions from the isocyanate functions leading to the vaporization of these compounds ${ }^{37-39}$.
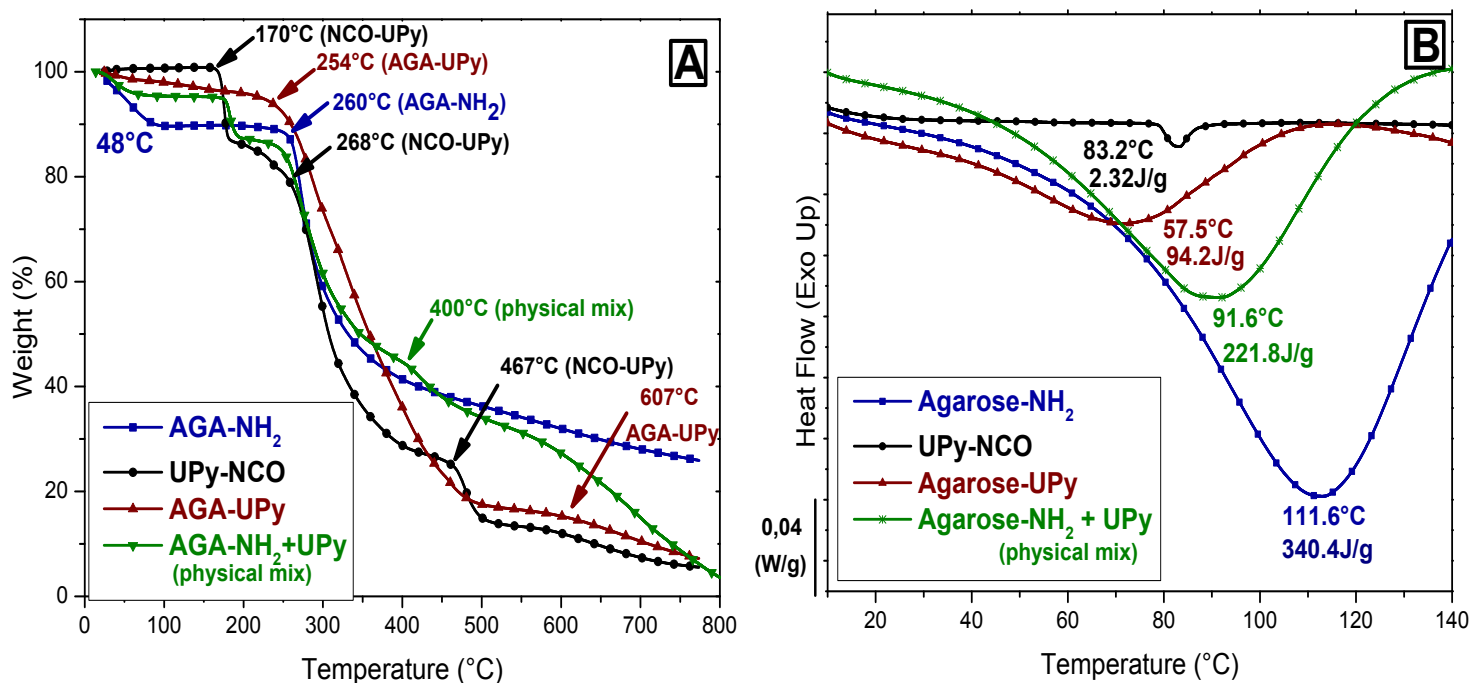

Figure 4. (A) Weight loss as a function of temperature (TGA) and (B) DSC thermograms:

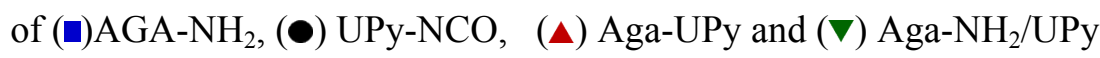

(Heating ramp $10{ }^{\circ} \mathrm{C} \cdot \mathrm{min}^{-1}, \mathrm{~N}_{2}$ atmosphere).

In order to confirm the reaction between the amine-agarose $\left(\mathrm{AGA}-\mathrm{NH}_{2}\right)$ and UPy-NCO compound leading to the formation of urea functions (AGA-UPy) as previously demonstrated by FTIR, the thermal stability of a physical mixture of AGA$\mathrm{NH}_{2} / \mathrm{UPy}-\mathrm{NCO}$ and AGA-UPy was studied by TGA. The thermograms clearly show that the degradation of the physical mixture of the two compounds retains the same degradation profile of the two initial compounds with the presence of characteristic decomposition peaks of $\mathrm{UPy}-\mathrm{NCO}$ and $\mathrm{AGA}-\mathrm{NH}_{2}$, respectively. On the opposite, AGAUPy presents a single degradation peak at around $360^{\circ} \mathrm{C}$ highlighting its higher thermal stability compared to UPy-NCO and AGA-NH2 . Moreover, AGA-UPy starts to degrade at $260{ }^{\circ} \mathrm{C}$ corresponding to the degradation of urea functions. According to the literature, Armstrong et al. have investigated the thermal behavior of UPy model compound based on a dimer of $\mathrm{N}-[($ butylamino)carbonyl]-6-methylisocytosine by gas 
chromatography-mass spectroscopy ${ }^{40}$. They have demonstrated that the degradation of butane-1-isocyanate occurs between $225^{\circ} \mathrm{C}$ and $245^{\circ} \mathrm{C}$ while the degradation of N,N'"di-n-butylurea takes place between $250{ }^{\circ} \mathrm{C}$ and $300{ }^{\circ} \mathrm{C}{ }^{40}$. Since AGA-UPy does not show any mass loss between $220^{\circ} \mathrm{C}$ and $245^{\circ} \mathrm{C}$, these results highlight that all the isocyanates functions of UPy have reacted with the amino group of AGA-NH2. In conclusion, the thermogravimetric analysis confirm the functionalization of aminated agarose by UPy leading to a thermally stable Upy modified-agarose.

The thermal behavior of UPy-NCO, AGA- $\mathrm{NH}_{2} / \mathrm{UPy}-\mathrm{NCO}$ physical mixture, and agarose functionalized with ureidopyrimidinone (AGA-UPy) were also investigated by DSC. As can be seen in Figure 4-B, a single endothermic peak around $83{ }^{\circ} \mathrm{C}$ with an enthalpy of $2.3 \mathrm{~J} / \mathrm{g}$ was observed for the synthesized monofunctional ureidopyrimidine (UPy-NCO). This phenomenon highlights a transition from mesophase to the isotropic state. UPy-dimers are capable of establishing H-bond in an orientational order forming a line joining the centers of nearest-neighbor molecules resulting in a nematic mesophase 41. In fact, many authors have widely reported the appearance of this endothermic peak by DSC for mono- and bifunctional UPy-NCO 10,16, 42. For example, Hirschberg et al. have shown that supramolecular aggregates based on UPy quadruple H-bonding built homeotropic monodomains in the liquid-crystalline state. As a consequence of this interaction, they observed a phase transition between $90{ }^{\circ} \mathrm{C}$ and $242{ }^{\circ} \mathrm{C}$ due to the highly viscous isotropic melt of UPy-dimers ${ }^{10}$.

Regarding AGA-NH $\mathrm{N}_{2}$ AGA-UPy and AGA-NH $\mathrm{NH}_{2} / \mathrm{UPy}$ physical mixture, endothermic peaks at $112{ }^{\circ} \mathrm{C}, 92^{\circ} \mathrm{C}$, and $57^{\circ} \mathrm{C}$ were obtained, respectively. In addition, differences in the peaks area were observed as a reflex of their different ability to retain water as well as the strength of polymer-water interaction. Polysaccharides are wellknown to display excellent ability to be easily hydrated due to their strong interactions 
with water molecules ${ }^{43}, 44$. Despite the water evaporation during the rise in temperature, the enthalpies and temperature corresponding to the endothermic peaks should undergo changes as a consequence of the functionalization of agarose ${ }^{45}$. Thus, large and similar enthalpies are observed for AGA-NH $\mathrm{NH}_{2}(340 \mathrm{~J} / \mathrm{g})$ and AGA-NH $\mathrm{H}_{2} / \mathrm{UPy}-\mathrm{NCO}(222 \mathrm{~J} / \mathrm{g})$ provide that the amount of mass introduced at the beginning is taken into account. In both cases, these results are consistent with the literature where the presence of amine ($\left.\mathrm{NH}_{2}\right)$ and quaternary ammonium $\left(-\mathrm{NH}_{3}{ }^{+}\right)$in the polysaccharide structure induced an increase in the amount of bound water ${ }^{46}$.

Otherwise, for AGA-UPy a significant decrease in enthalpy $(94 \mathrm{~J} / \mathrm{g})$ and a shift to lower temperatures $\left(57^{\circ} \mathrm{C}\right)$ may also be observed indicating a disappearance of amine functional groups. This event can be explained by the formation of urea functions issue from the reaction between the isocyanate functions of UPy-NCO and the amine functions of agarose. In addition, the decrease in the exothermic peak temperature (57 ${ }^{\circ} \mathrm{C}$ instead of $112{ }^{\circ} \mathrm{C}$ ) confirms the agarose hydrophobization characterized by the disappearance of the presence of bound water. In conclusion, the study of different functionalized agaroses by their thermal behavior shows an increase in the hydrophobicity of the agarose due to its functionalization by the monofunctional ureidopyridimidinone (UPy-NCO) compound.

\subsection{Effect of Crosslinking Dimerization}

Multi-functional materials displaying supramolecular interactions are wellknown as dynamic materials and are inspired by biological systems due to their easy adaptability. Increasingly, different classes of interactions have been investigated to design materials based on crosslinked physical interactions such as hydrogen bonds ${ }^{47}$, 
ionic ${ }^{48}$, host-guest recognition ${ }^{49}$. Recently, H-bonding as well as weak hydrophobic interactions were shown to improve the stability of physically cross-linked hydrogels formed by telechelic PEG-hydroxylase enzymes ${ }^{50,51}$. On the basis of these perceptions, we synthesized AGA-UPy which was mixed with unmodified agarose at different ratios to reduce the brittleness of this gel from non-covalent agarose backbone interactions. Agarose was selected owing to its excellent water solubility, excluding the requirement for organic solvents. More importantly, its fast rate of gelation ensures the different modeling of these non-cytotoxic 3D hydrogels and efficient encapsulation of active principles, growth factors, cells and so on. On the other hand, the human and animal cells can not adhere to the polysaccharide due to the formation of a rigid, hydrophilic, and inert hydrogel. The introduction of hydrophobic- and H-bonding interactions into polysaccharide hydrogels is expected to reproduce the natural environment of cells, which provide gels the capability to flow ${ }^{52}$. In light of designing materials that are simple to prepare and process for cell scaffolds, we were inspired by H-bonds of UPy to design a hydrogel and subsequently supramolecular sponges-like scaffold capable of absorbing and fixing biological fluids such as cells, growth factors and culture medium. 

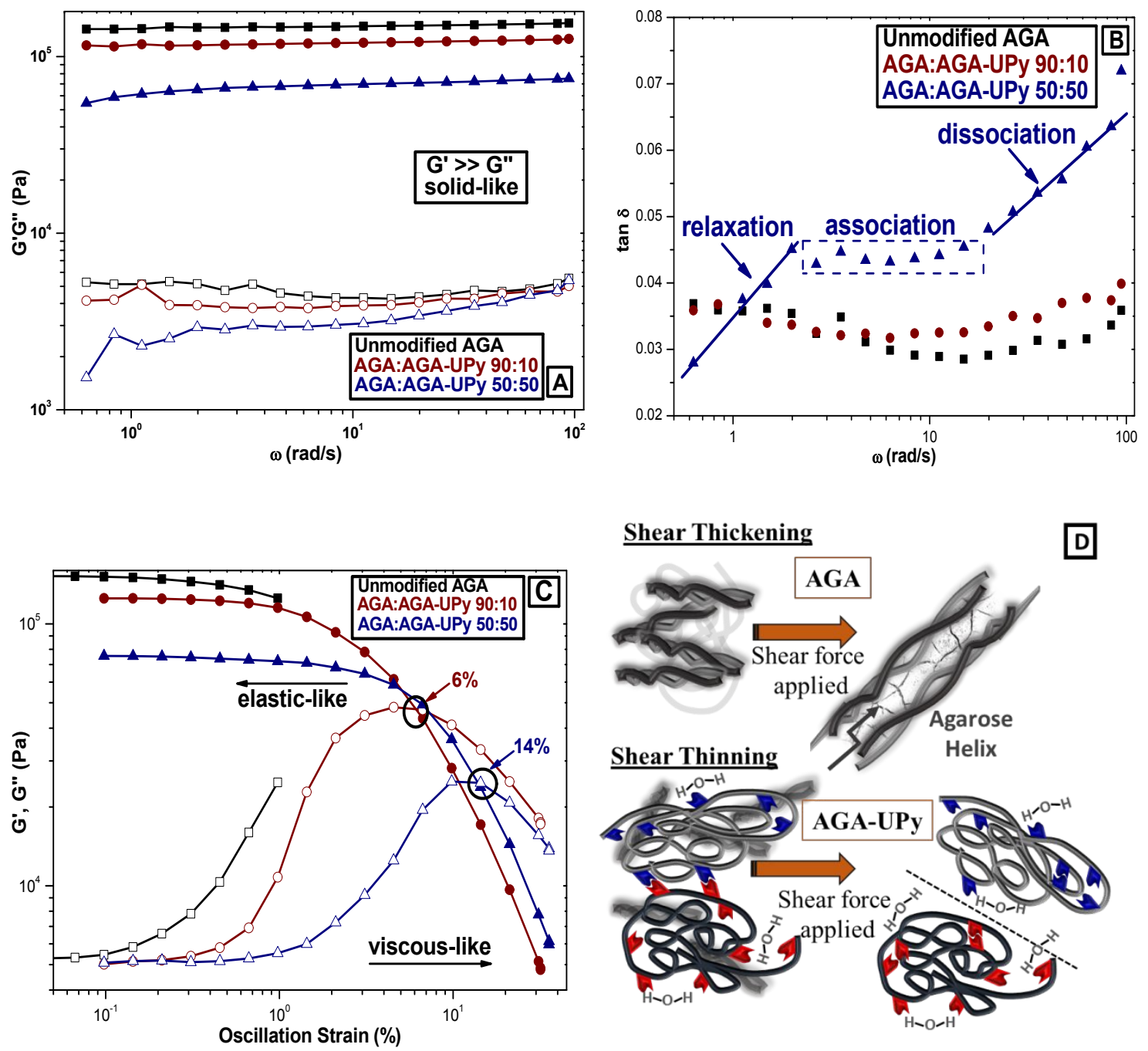

Figure 5. (A) Frequency dependence of G' (full symbols) and G" (empty symbols) for AGA and AGA:AGA-UPy 90:10 and 50:50, (B) $\tan \delta$ dependence in frequency sweep, (C) Strain sweeps in oscillatory shear $\left(5 \mathrm{rad} \mathrm{s}^{-1}\right)$ showing nonlinear dependence, G' (full symbols) and G" (empty symbols), and (D) Schematic representation of possible shear effects on AGA-UPy hydrogels, and representations of shear thickening aggregates and flow aligned shear thinning aggregates (UPy dimers are represented in the figure by the different colors red and blue).

The hydrogels were prepared from a hot aqueous solution and for all compositions the gelatinization was verified by visual observation arisen in a few seconds at $37{ }^{\circ} \mathrm{C}$. For all samples, under oscillating shear for all samples (Figure 5-A), 
the storage modulus $\left(\mathrm{G}^{\prime}\right)$ of the hydrogels was found to be far higher than the loss modulus $\left(G^{\prime \prime}\right)$, indicating solid-like behavior. Frequency sweep experiments revealed that hydrogel with $50 \%$ of AGA-UPy (50:50) exhibits frequency dependent behavior with nonlinear increase of $\mathrm{G}^{\prime}$ and $\mathrm{G}^{\prime \prime}$, which is a characteristic of dynamic interaction in gels ${ }^{50}$.

Likewise others classical supramolecular counterparts, a non-linear behavior is observed for the sample with $50 \%$ AGA-UPy in Figure 5-B. A characteristic of supramolecular networks when oscillation frequencies increase is that their dynamics is driven by different timescales; just as relaxation of supramolecular interactions, followed by organization/association of supramolecular interactions, and then breakage of supramolecular associations or inter-chain crosslink ${ }^{53}$. The networks with higher amounts of UPy (50:50) show firstly a chain relaxation, a plateau at frequencies between 2 and $20 \mathrm{rad} / \mathrm{s}$, then bonds-dissociation upon increasing frequency. This association effect occurs due to the organization of the chains owing to force application, producing a transient state. Afterwards, a breakage of supramolecular bonds occurs and the gel moves to a viscous-fluid state. Similarly, Li et al. presented that macroscopic viscosity and shear behavior of a low molecular weight dye in polymer melts are oriented by association and dissociation of H-bonding motif in UPy functional group ${ }^{54}$. Those authors prepared reversibly associating copolymers containing UPymotif and reported that stress relaxation is therefore dominated by the rate of UPy association-dissociation.

Figure 5-C displays the strain dependence of the elastic and loss modulus for hydrogels, measured at an oscillation frequency $f=15 \mathrm{~Hz}$. At the lowest strains the response is solid-like, with a $G^{\prime}$ value that is greater than the $G^{\prime \prime}$, while at the highest frequencies the loss modulus dominates the response for samples with AGA-UPy 
(50:50 and 90:10) indicating viscous-like behavior. A striking feature of supramolecular networks is that as the strain increases at fixed $\omega$, the loss modulus initially upsurges to a peak before finally decreasing at the largest strains indicating that dissipative processes occur ${ }^{53}$. This behavior may be elucidated as a consequence of a slow structural $\mathrm{H}$-bonding relaxation process that is predictable in dimers-interaction at very low strain deformation. Instead, unmodified agarose hydrogel displays no liquid-like characteristics showing a brittle behavior, breaking a rheological yield strain at $1 \%$. However, the hydrogels with $10 \%(90: 10)$ and $50 \%$ (50:50) retain an overall solid-like behavior, with $\mathrm{G}^{\prime}$ superior than $\mathrm{G}^{\prime \prime}$ and only feebly dependency on $\gamma(\%)$. Hereupon a few percent of applied strain amplitude, $G^{\prime}$ decrease significantly and G" grows until G' $=\mathrm{G}^{\text {" at a 'fluidization' strain }}{ }^{55}$. Beyond the critical strain, both moduli drop with strain, but the G" dominates indicating a fluid-like behavior. The critical strain crossover for samples with $10 \%$ UPy (90:10) is $\gamma=6 \%$, while for hydrogels with $50 \%$ of AGA-UPy (50:50) the fluidization crossover occurs at $\gamma=14 \%$ due to the greater dimer interaction. Hydrogels with H-bond-crosslinked supramolecular networks (UPy) showed the ability to switch between solid-like and liquid-like states, belonging to the family of shear-thinning hydrogels ${ }^{56}$. The shear-thinning is attributed to networks with rapidly associating-dissociating disentanglements or fast decrosslinking kinetics ${ }^{53,57}$. When the linkage chain relaxation arises quicker than the reconnection of supramolecular interactions, shear-thinning appears due to the transformation of intramolecular to intermolecular bonds, as shown in Figure 5-D. Similar results have been achieved by Lehn et al. for hydrogen-bonded supramolecular networks associated with an intense decrease of the viscosity of semidilute solutions ${ }^{58}$. In the opposite case, hydrogels with only unmodified agarose showed shear-thickening feature, where intertwined helices chains become more stressed when submitted to mechanical stress. On the macroscopic 
scale, the effect of shear-enhancement in shear-thickening hydrogels is a fracture resulting in the brittle and stiff materials. Accordingly, the used supramolecular dimers with crosslinked domains was seen to increase the gel strain capacity to high deformation, leading to gels with superior energy dissipation and enhanced resistance to shear deformation.

To further confirm the supramolecular dimers interactions, SANS measurements were performed and the following functional form is fitted to the data (Figure 6). We quantitatively evaluated the SANS patterns by Beaucage model, where the unified equation with two terms is used in order to describe a complex morphology in a wide range of $\mathrm{I}(\mathrm{q}){ }^{59}$. The correlation lengths $(\xi)$, radius of gyration $\left(\mathrm{R}_{\mathrm{g}}\right)$ and fractal dimension $\left(\mathrm{D}_{f}\right)$ in hydrogels were determined by equation 3 . The first term of equation is an Ornstein-Zernike function that describes the polymer concentration fluctuation ${ }^{60}$. The second term is described by Guinier's law, which describes excess scattering from a spatial inhomogeneity ${ }^{61}$.

$$
I(q)=\frac{1}{1+q^{2} \xi^{2}}+\exp \left(\frac{-q^{2} R_{g}{ }^{2}}{3}\right)
$$

Because agarose chains are able to form mechanically robust hydrogels, AGAUPy hydrogels scattering contributions show a microphase separation behavior from the UPy-dimers, driven by hydrogen-bonds and hydrophobic interactions. The introduction of physical crosslinkers intrinsically leads to regions which are less accessible to water. According to Mihajlovic and co-workers, the physical crosslinker junctions for the UPy-dimers produce spherical inhomogeneities as nano or microdomains ${ }^{52}$. These dimers act as crosslinking points that are bridged and surrounded by agarose chains forming supramolecular aggregates, as shown schematically in Figure 6. 

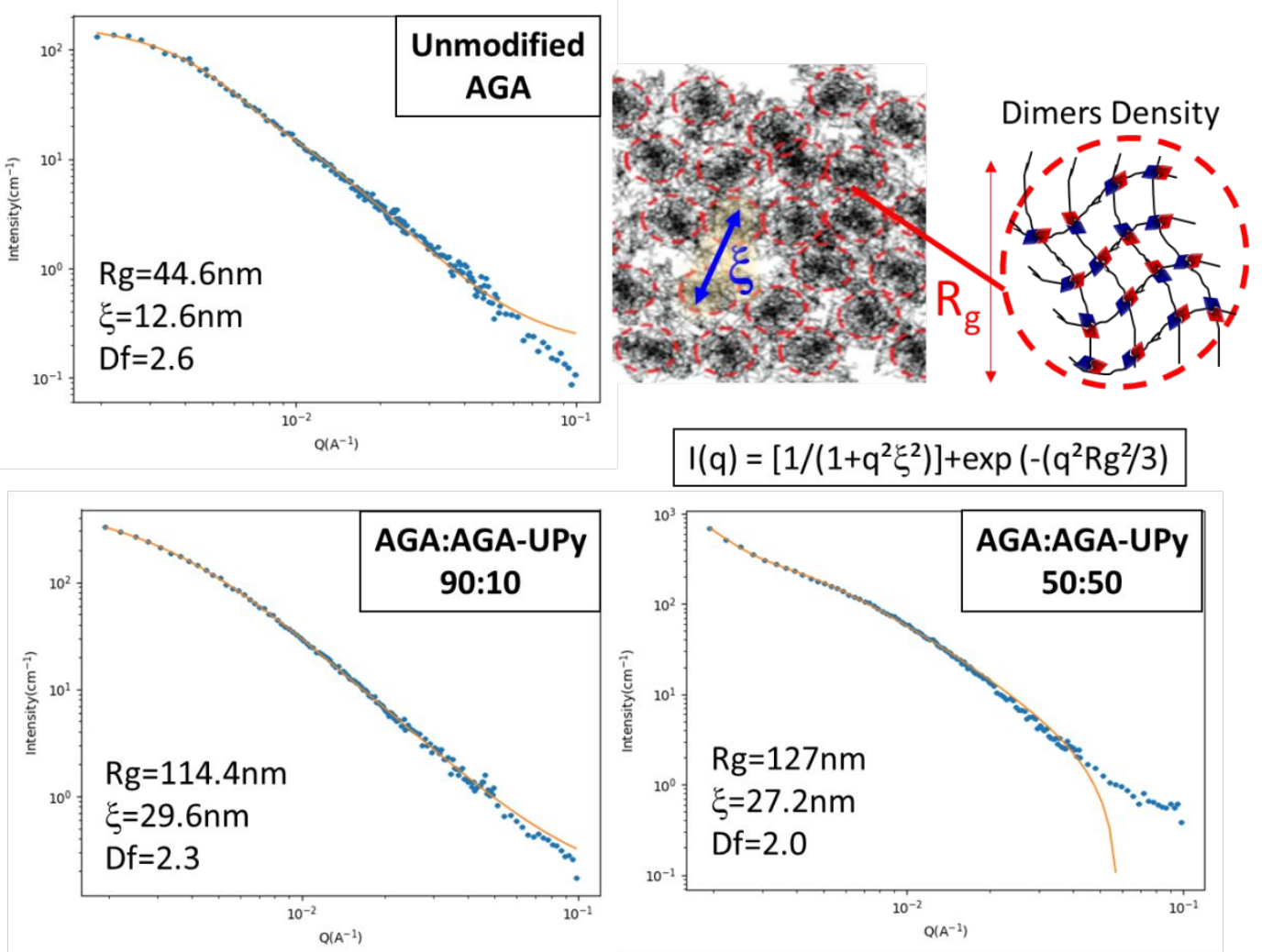

$$
I(q)=\left[1 /\left(1+q^{2} \xi^{2}\right)\right]+\exp \left(-\left(q^{2} \operatorname{Rg}^{2} / 3\right)\right.
$$

Figure 6. Schematic of supramolecular network effect on the polymeric aggregates (UPy dimers are represented in the figure by the different colors red and blue).

Mainly in hydrogels with $50 \%$ AGA-UPy (50:50), due to a great dimers fraction used, the correlation peak is much more pronounced because the phase separation is more distinct. Thereby, we managed to study morphology of the aggregates as well as their correlation distances. The influence of dimers on the formation of supramolecular aggregates can be illustrated by the $\xi$ values. For unmodified agarose, the $\xi$ value is 12.6 $\mathrm{nm}$, while the incorporation of UPy-dimers doubled it. The increased of correlation length in $10 \%$ AGA-UPy (90:10) arises from the interaction between dimers and can be associated to the medium spacing between the hydrophobic domains or dimer aggregates, which reduces in $50 \%$ AGA-UPy (50:50) and becomes more defined upon 
an increase in dimer volume fraction (e.g. $\xi=29.6 \mathrm{~nm}$ for AGA:AGA-UPy 90:10 versus $\xi=27.2 \mathrm{~nm}$ for AGA:AGA-UPy 50:50). The formation of these supramolecular aggregates has also the consequence of increasing the radius of gyration, we can observe values of $\mathrm{Rg}=44.6 \mathrm{~nm}, 114.4 \mathrm{~nm}$, and $127.00 \mathrm{~nm}$ for unmodified-AGA, AGA:AGA-UPy 90:10, and AGA:AGA-UPy 50:50, respectively. This Rg variation agrees with the results obtained in rheology and the scheme shown in Figure 5-D.

The fractal dimension $\left(D_{f}\right)$ in hydrogels may be detected for either surface or mass fractals. Experimentally, information illustrating the aggregate sizes and structures of the hydrogels by SANS may be acquired by quantifying the density gradient of the hydrogel ${ }^{62}$. The Guinier equation provides the fractal dimension of the network structure, $\mathrm{D}_{f}$ values ranging between 2.0 and 2.6 inferring the formation of dense network domains ${ }^{63}$. This behavior is credited to the interface between the aggregates. As expected, $\mathrm{D}_{f}$ decreases with increasing $\mathrm{H}$-bonding interaction as the dimers volume fraction in the gel is greater. To sum up, SANS and rheology measurements revealed the aggregation into connected structures from dimers interactions.

\subsection{Sponges-like scaffold formation and their architecture}

It is common understanding that a three-dimensional setting with a suitable microstructure is crucial to promote pre-vascularization for oxygen exchange driving tissue regeneration, as well as to protect cells from mechanical stress. Besides, the ability to absorb and retain biological fluids in sponge-like scaffolds leads to material properties which are comparable to some physic-chemical properties of soft tissue. Hydrogels obtained by the sol-gel process are probable candidates to be turned into sponges by freeze drying in order to promote a vascularized cell support. These 
hydrogels should be able to avert the pore collapse phenomenon retaining the porous texture of the wet material ${ }^{64}$. However, the development of many hydrophilic polymerbased hydrogels, and subsequently sponges, for cell growth and tissue regeneration, sometimes is not achievable because of their poor cell attachment properties.

Taking this into account, we report a structurally new type of sponges, supramolecular sponges, derived from hydrogels formed by homo-complimentary hydrogen bonding. The UPy-dimers were used as an alternative supramolecular crosslinker to modify the microstructure and surface of the sponge. Hydrogels with AGAUPy provide the same rate of rapid gelation of agarose hydrogels as scaffold, which allowed us to analyze the influence of microstructure on cell attachment within the three types of sponges: unmodified agarose, and sponges with $10 w t \%(90: 10)$ and $50 w t \%$ (50:50) of synthesized AGA-UPy. The hypothesis was that these different amounts of supramolecular crosslinking would result in a great difference in sponge microarchitecture (Figure 7), hence this difference will alter the attachment of neural stem cells cultured in the scaffolds.

As mentioned in the previous section, the unmodified agarose and synthesized AGA-UPy conjugate were mixed; then hydrogels were rapidly generated within few seconds by simply cooling the solutions. All the cubical hydrogel samples were prepared inside plastic capillaries (with an internal diameter of $1 \mathrm{~cm}$ ). The unique structure of sponge scaffold-like has been generated by removal of the liquid component of gel by freeze-drying. The fast-freezing kinetic leads to a greater nucleation rate of ice crystals and consequently to vast number of small crystals. In the conventional freezing method the freezing rate is slower favoring crystal growth, thereby a larger pore size is reached ${ }^{65}$. In order to obtain a great porosity and firmer polysaccharide walls, we chose to produce sponges of agarose crosslinked with supramolecular UPy to compare with 
unmodified agarose by the slow freezing $(24 \mathrm{~h})$ and conventional freezing-dry methods (24 h). This method suggests the creation of ice crystals in the microscopic spatial sphere (Figure 7) and the formation of completely random polymer walls. Followed by freezing-drying process, the water elimination yields micro-sized pores, resulting in sponges-like materials.

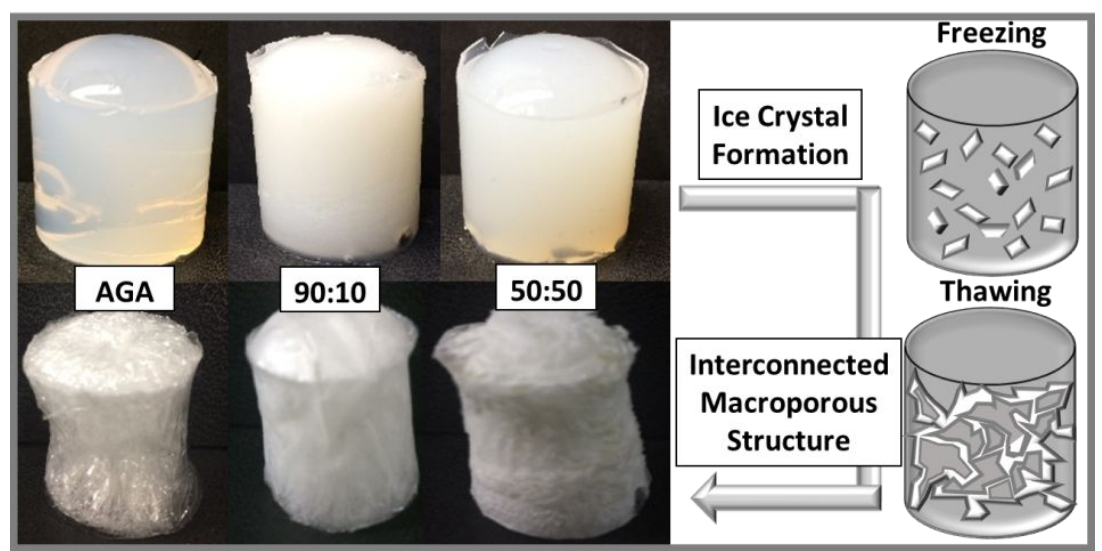

Figure 7. Fabrication of sponges which results in the formation of a macroporous, marshmallow-like structure.

Macroscopically, all sponges show a three-dimensional (3D) marshmallow-like shape. However, we can observe that a more dense structure in aerogel with $10 \%$ AGA-UPy is formed. The microstructure of highly porous supramolecular AGA-UPy sponges was investigated via transmission electron microscope tomography and SEM images (Figures S6). A series of images was then acquired, segmented, and reconstructed in order to create a three-dimensional (3D) model of the aerogel (Figure 8). X-ray microtomography confirmed the 3D structure, monolithic and porous structure. The stacks of two-dimensional XMT images from the top of sponges are shown in Figure 8. These images visibly show colorful walls corresponding to the AGA and AGA-UPy solid structure and black areas consistent to the air voids. Sponges-like 
materials displayed the anisotropic structures of the pores that may easily be seen when the sample is illustrated along different axes (XY-axis and $\mathrm{XZ}$-axis).

The sponges show an sophisticated open cellular architecture of interconnected pores. In all samples, especially the sample with unmodified AGA, the polymer chains have been collapsed to form a sheet-like structure during the sublimation process. Physical significance to determine the porosity in sponges is unclear because real pores may have different geometry. Conventional freezing process causes a random growth of ice crystals and, as a consequence, an irregularity in the pore structure. However, we found that a smaller porosity was formed in unmodified agarose sponges $(87 \% \pm 0.44)$. Observing the area around the pores, we can noticed that sponges prepared from unmodified agarose are formed of walls with different thicknesses ranging from $24 \mu \mathrm{m}$ to $88 \mu \mathrm{m}$. Sponges with $10 \%$ AGA-UPy supramolecular crosslinking possess thinner walls, thicknesses with a non-homogeneous size distribution, and a slight increase in porosity, $90 \%$ of porosity $( \pm 0.96)$. The pores for these sponges (90:10) are also anisotropic similar to unmodified sponges, but smaller. The structures are a highly interconnected network and the pores are heterogeneous in shape and size (watch the video on SI, 90_10). When the amount of AGA-UPy increases, the wall thickness of sponges became homogeneous and the sponges lie in mesoporous range. The presence of strong hydrogen bonding of UPy-dimers may cause greater interaction with water and a better distribution of the chains. Thereby, when the agarose sponges were reinforced with $50 \%$ of supramolecular AGA-UPy a remarkably change in wall structure can be noticed. Most of wall thicknesses for this sponge (50:50) are in the range of $24 \mu \mathrm{m}$ to $40 \mu \mathrm{m}$ and the porosity increases to $92 \%( \pm 0.47)$. Indeed, the sponges obtained from agarose with $50 \%$ AGA-UPy (50:50) did not show external volumetric shrinkage (watch the video on SI, 50_50). In unmodified agarose sponges, 
the wall is thickness and random because of the stiffer structure of the agarose hydrogels. Therefore, reinforcement by AGA-UPy resulted in a better distributed and flexible structure.

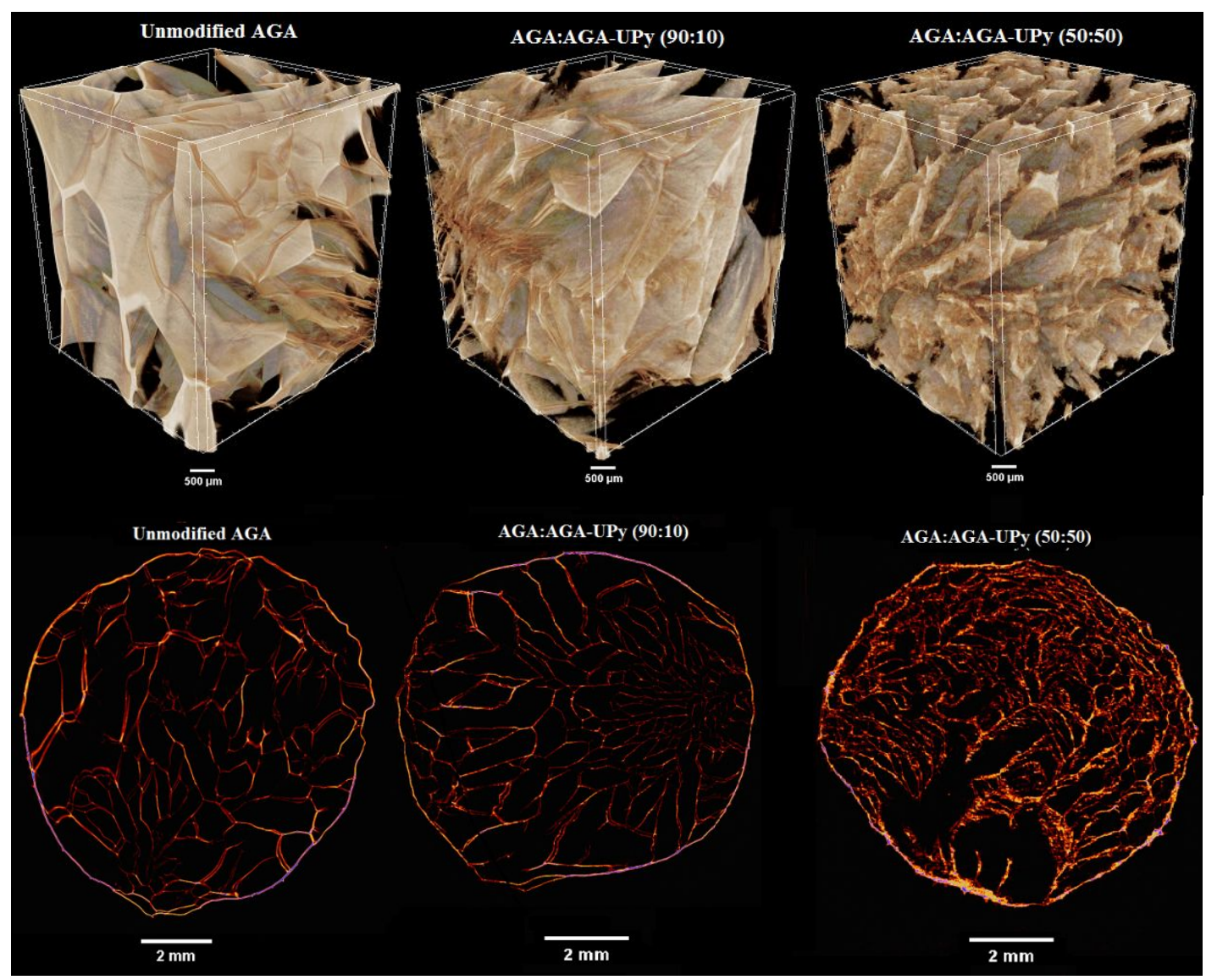

Figure 8. Three-dimensional reconstructed images from microtomography of aerogels $(5 \% \mathrm{w} / \mathrm{w})$ : neat agarose aerogel, agarose aerogel with $10 \%$ AGA-UPy $(90: 10$ of AGA:AGA-UPy), and agarose aerogel with $50 \%$ AGA-UPy (50:50 of AGA:AGAUPy). Two-dimensional reconstructed images from microtomography.

Regardless the proportion of AGA-UPy used in the sponge production, the density and linear shrinkage for all sponges did not change significantly during the freeze-drying process. As mentioned above, unmodified agarose sponges are also 
porous and show low-density structure, but the major drawback is that these sponges are incompressible and extreme brittle. The mechanical strength of sponges-like scaffolds is a very important parameter for cell storage and transportation. Supramolecular crosslinking by UPy molecules can sideline these drawbacks improving the malleability, compressibility, and flexibility without compromising their original properties. The purpose of adding supramolecular dimers to the material is to enhance the intra and intermolecular interaction of the polysaccharide backbones. Therein, mechanical properties of sponges were evaluated by analysing their microstructure network. The sponges with AGA-UPy can bear compression strain of $45 \%$ without permanent deformation or mechanical destruction (Figure S5). By contrast, strain of unmodified agarose sponge increased as stress increased; this caused greater internal damage and thus slower recovery. The stresses of the sponge were about $50 \mathrm{kPa}$ at $45 \%$ strain for samples with AGA-UPy and $5 \mathrm{kPa}$ at $35 \%$ strain for unmodified agarose in the first cycle (Figure S5). Such higher strain of crosslink supramolecular demonstrates the elastic nature of the scaffold and thereby supports its use to culture of neural stem cells (NSCs). The soft and elastic nature of the synthesized AGA-UPy sponges provides an appropriate physical property for neural tissue engineering.

Water uptake is another crucial factor for nutrient and metabolite transport in cell scaffolds. It is also crucial to determine the ability of the sponges to retain biological fluids and cells adhesion. To examine whether this has not been compromised due to the addition of UPy dimers, the water uptake properties of the supramolecular sponges were compared to those of unmodified sponges. In terms of physical properties, there was no significant change in sponges swelling using different UPy concentrations. Unmodified agarose sponges were found to maintain water up to thirty times their own weight. Moreover, there was no major difference in water 
retention for sponges after the addition of AGA-UPy (26 times). The higher UPy concentration (50:50) yielded lower water retention, which demonstrates their hydrophobicity (11 times). Despite their lower water retention capacity, sponges with 50 \% AGA-UPy show a good balance between more hydrophobic interfaces (desirable for cell adhesion) and adsorption capacity of biological fluids, such as proteins, culture medium, cells and growth factors.

\subsection{Supramolecular dimers effects on Neural Stem Cell Adhesion}

Neural stem cells (NSCs) play a therapeutic role in neurodegenerative diseases and may contribute to functional recovery. Upon transplantation, NSCs substitute lost cells and throw molecules that stimulate the survival of the remaining local cells. Nonetheless, neural-based therapies still face several obstacles as the majority of cells die post-allogeneic transplantation 2,66 . To circumvent this problem, earlier studies have been developed to yield good 3D substrates for neural tissue engineering, such as biodegradable, bioresorbable, and piezoelectric scaffolds ${ }^{1,2}$. Herein, 3D substrates with supramolecular UPy-dimers were assessed as support for NSCs. As described above, we have developed an efficient sponge-like scaffold capable of rapidly absorbing cells, growth factors, and culture medium into the supramolecular-crosslinked biomaterial. These sponges after the immediate incorporation of cells and culture medium turn into an elegant shear-thinning hydrogels.

Upgrading cell adhesive potential of non-adhesive scaffold is an important goal in neural tissue engineering. It is well-known that neuronal cells interact with the substrate through adhesion molecules, wherein integrin receptors form a major class of proteins that mediates cell adhesion into extracellular matrix (ECM) ${ }^{67}$. Integrins 
receptors are transmembrane $\alpha-\beta$ heterodimers from members of the large $\alpha$ and $\beta$ gene families intricate in the regulation of survival, proliferation, migration, and differentiation ${ }^{67-69}$. In mammals, integrins are composed of $8 \alpha$ and $8 \beta$ subunits that heterodimerize to form 24 unique receptors ${ }^{70,71}$. In neural cells, these integrins receptor engagement and clustering is controlled by ligands from the ECM (or scaffold, in the case) leading to the formation of focal adhesions. Therefore, molecules of interest in scaffold can help create a better connection between integrin receptors and surfaces, which are able to promote actin filaments in cells ${ }^{6,72}$. Biomaterials can be modified with molecules-recognition motifs to enhance neural tissue integration and promote ECM production. Towards this end, the inclusion of supramolecular UPy-dimers into the sponges has the dual objective of improving the mechanical properties, while simultaneously incorporating adhesion segments. UPy-dimers represent here one class of adhesion molecules that can promote a better neural cells attachment. These adhesion properties in scaffold mimic the microenvironment of natural neural tissue resulting in better cell behavior.

In order to evaluate the cell affinity and attachment in supramolecular-dimers scaffolds, NSCs were seeded into the sponge-like scaffolds and cultured for 3 days. In previous work, we have already demonstrated that primate NSCs exhibit high proliferation rate and can be expanded continuously in long term culture ${ }^{1,18}$. These primate NSCs express SOX2, a classical marker of NSCs, as well as the proliferative marker, Ki67. In this study, the immunofluorescent analyses (Figure S7) show that the vast majority of NSCs cultured in Neat AGA and AGA modified with UPy expresses SOX2, suggesting that sponges-like scaffolds sustain the proliferation and maintenance of primate NSCs. We also took advantage of our NSCs expressing TAU-tagged GFP, which labels microtubule components of the cytoskeleton, including long cell processes 
and other subcellular structures, thereby enabling fine visualization of cell morphology. Complementary staining of Filamentous-actin (F-actin) allowed finely detailed visualization of interactions between cells, as well as between cells and scaffold.

Confocal microscopy images after cell seeding, allowed us to evaluate cells distribution and attachment into scaffolds (Figure 9, blue channel=cells-dapi and green channel=GFP). From fluorescence images in Figure 9-A and 9-B, it is clear that NSCs seeded into unmodified agarose sponge exhibit a poor contact with the scaffold surface. Cell images on these sponges show that cell-cell adhesion is stronger than scaffold adhesion, forming clusters. The initial cell adhesion on surfaces is crucial for survival and growth, as the attachment determines cytoskeletal organization, cell shape, motility, proliferation and differentiation ${ }^{73}$. As a result of this poor attachment we can observe in Figure S8-D and -E the DNA defragmentation, the absence of actin fibers, and consequently, the death of most cells into unmodified agarose scaffold. On the other hand, NSCs into sponges with $50 \%$ AGA-UPy (50:50) there are more contact areas between the cells and scaffold surfaces to which the cells can attach (red arrows in Figure 9-C and -D). Instead, into $10 \%$ AGA:UPy sponges (90:10) the cells concentrate around the AGA:UPy aggregates (yellow arrows). The images of Figure 9-D and -F show that cells attach around the AGA-UPy surfaces with a typical NSC morphology. Due to the greater amount and better distribution of AGA-UPy into sponges, we observed a spread-out distribution of NSCs along the scaffold surface and throughout scaffold (Figure S8-B). Whereas, in an overview of the throughout unmodified agarose scaffold a large void space was observed with a sporadic cells distribution (Figure S8A). In order to better show the integration of the cells in three dimensions, $\mathrm{z}$-stack images were taken at $3 \mu \mathrm{m}$ intervals through the scaffolds (Figure S9), which show that cells infiltrate within AGA:UPy scaffolds establishing contacts at different levels in the 
deepness of the scaffolds (indicated by the red arrows). In contrast, very few contacts were observed between the cells and Neat agarose.

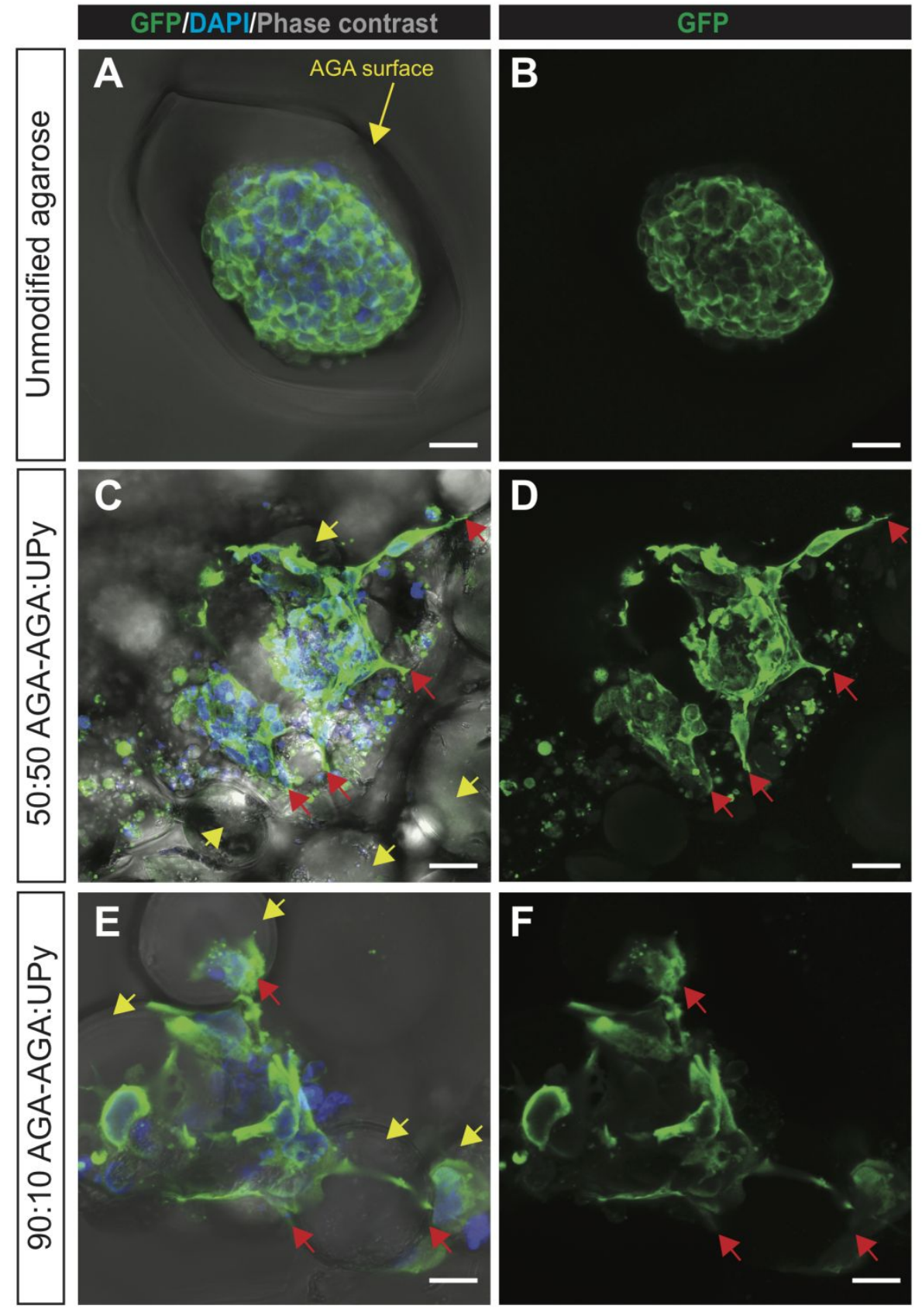

Figure 9. Integration of NSCs in sponge-like scaffolds. Confocal images of NSCs following culture in (A, B) unmodified agarose; (C, D) 50:50 AGA-AGA:UPy; (E, F) 90:10 AGA-AGA:UPy. (A,C,E) Merge images of nuclei stained with DAPI (blue), GFP (green), and phase contrast images (grey). (B,D,F) GFP staining. Maximum intensity projections of z-stack images are shown. Yellow arrows indicate AGA-UPy aggregates; red arrows indicate cell-scaffold interactions. Scale bars, $10 \mu \mathrm{m}$. 
As above-mentioned, cells request an essential adhesion area on a substrate to survive, and the nature of this contact area may create the formation of associates with the outside environment 6,74 . The development and proliferation of neural cells in suitable scaffold usually follows the following steps: (1) preliminary cell attachment, (2) cell spreading, (3) organization of the actin cytoskeleton, and (4) formation of specific focal contacts ${ }^{6}$. Actin organization and focal adhesions mobilization are driven by a grouping of extrinsic and intrinsic signals, counting extracellular matrix proteins, growth factors, and extracellular molecular protagonists, such as neural cell adhesion molecules ${ }^{75}$. UPy dimers can mediate $\alpha-\beta$ heterodimerization for the neural cells attachment via a non-receptor-mediated cell link mechanism, i.e., from its quadruple hydrogen-bonded system, hydrophobic interactions or by its functional amine groups ${ }^{76}$. The UPy moieties provide reactive amine groups allowing site-specific peptide immobilization using cysteine terminated peptides or interaction with amino-terminal domain contained in the nucleotide-binding region ${ }^{77-79}$. These chemoselective moieties can interact with specific integrin peptides in order to preserve receptor recognition, which is reliable with published results on the nonspecific interaction between amine functionalized materials and cell $7,77,78$ and more specifically neurons ${ }^{6,79}$. This outward signal transfer can produce an induced redistribution of integrin in the cell membrane, resultant in a 'cell-scaffold interaction' comparable to the ligand-receptormediated interaction and ECM molecules, such as vitronectin, laminin, collagen or fibronectin ${ }^{6}$. Beyond that, the incorporation of specific bioactive molecules can also improve adhesion indirectly, by endorsing the adsorption of ECM proteins through hydrophobic interactions ${ }^{79,80}$. This adsorption can occur after the initial binding, induced by adhesive molecules, when cells secrete ECM and the mechanotransduction processes is released. It is important to highlight that cells incapable to secrete ECM are 
unable to sustain binding by this mechanism and undergo apoptosis ${ }^{6,80}$. Moleculemodified cell adhesion has already been employed in polysaccharides for neurite outgrowth, demonstrating the importance of adhesion molecule selection when creating scaffolds. For example, covalently modified agarose with laminin-derived peptides was revealed to be larger to the original agarose hydrogels in endorsing neural adhesion and axonal growth ${ }^{9}$.

Following cell attachment to unmodified AGA, AGA-UPy 50:50, and AGAUPy 90:10, filamentous-actin was stained in order to visualize the interactions between cell-cell and cell-surface. Cell images of NSCs seeded on unmodified agarose scaffold show that cell-cell adhesion is stronger than scaffold adhesion, with cells forming densely packed cell clusters and a poor contact with the surface (Figure 10-A, -B, and C). For all samples with AGA-UPy, the NSCs actin appears to associate on UPy-dimers surfaces, as shown in Figure 10-D to -I. When the dimers association formed aggregates into sponges, it is possible to notice that the cells surround these aggregates showing several points of contact. The initial attachment of NSCs onto sponge surfaces modified with UPy-dimers results in immobilization, avoiding detachment in answer to slight shear forces. When the surface of the biomaterial is suitable for the cell attachment, the cell membrane begins to spread along the available surface area, as shown in Figure 10$\mathrm{H}$ and -I. This event is succeeding with the formation of a filamentous actin cytoskeleton. Finally, integrins form clusters in response to force applied by the cytoskeleton, known as focal adhesion sites. These trigger signaling trails can influence cell function, viability, and proliferation ${ }^{81}$. SEM images revealed NSCs morphology spreading on AGA-UPy surfaces (Figure 10-K and -L). Meanwhile, cells seeded on unmodified agarose sponges appear to repulse the surface (Figure 10-J). 

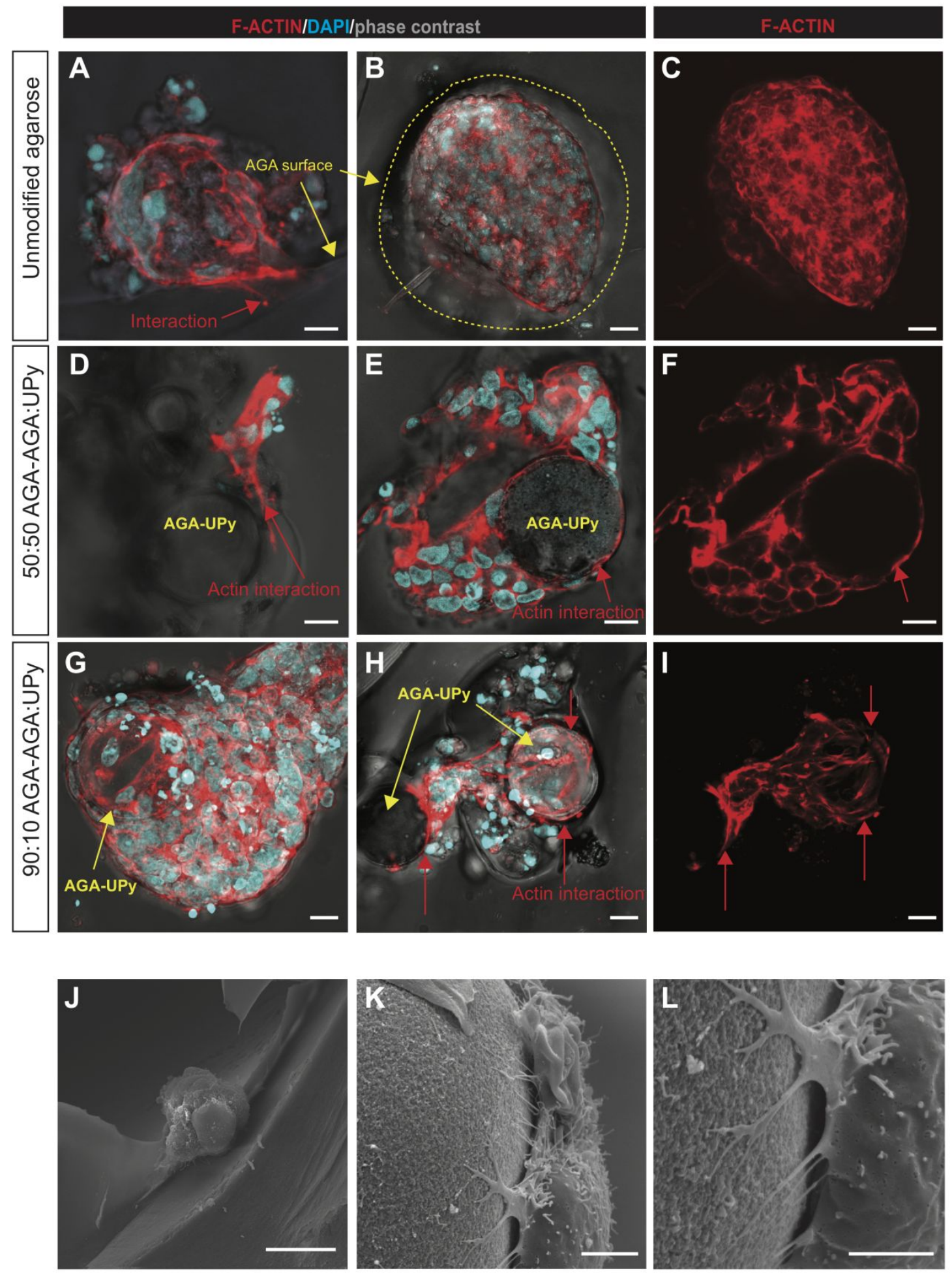

Figure 10. Interaction between NSCs and sponge-like scaffolds. (A-I), Confocal images of the sponge-like scaffolds following cell attachment, stained for F-Actin. (A, B, C) unmodified agarose; very few contacts are detected between the cells and scaffold surface (yellow dotted line); (D, E, F) 50:50 AGA-AGA:UPy. (G, H, I) 90:10 AGA- 
AGA:UPy. (A,B,D,E,G,H) Merge images of nuclei stained with DAPI (cyan), F-Actin stained with Phalloidin (red), and phase contrast images (grey). (C,F,I) F-Actin stained with Phalloidin (red). Maximum intensity projections of z-stack images are shown.

Yellow arrows indicate AGA-UPy aggregates; red arrows indicate cell-scaffold interaction. Scale bars, $10 \mu \mathrm{m}$. (J, K, L) Scanning electron microscope images of NSCs cultured on unmodified AGA (J) (10 $\mu \mathrm{m}$, mag 2000X), and 50:50 AGA:AGA-UPy (K, L) (Scale bars: $5 \mu \mathrm{m}$ and $10 \mu \mathrm{m}$, respectively; mag $8000 \mathrm{X}$ ).

Topology and mechanical compliance of the substrates are two other factors that may have contributed to the initial cells attachment. Surface properties of a scaffold have determinant effects on the NSCs behavior. As shown in Figure S6 (see support information), the surfaces of sponges-like scaffold with $10 \%$ and $50 \%$ of AGA-UPy show significant roughness and surface spreads with waviness and small droplets, while unmodified agarose sponge surfaces are very smooth. The surface roughness can improve cell adhesion due to increased contact area, which is related to the interfacial adhesive force ${ }^{82}$. According to Khan et al., increase contacts between cell body and scaffold surface improves subsequently the neural proliferation ${ }^{83}$. Furthermore, Decuzzi and Ferrari have shown that the roughness is contrariwise to the adherent cell stiffness, i.e., cells with less stiff membranes require more surface roughness to initiate their adhesion ${ }^{84}$. This hypothesis is in agreement with the results obtained here because the brain is a soft tissue, 10 times softer than liver and 50 times softer than muscle ${ }^{85}$. We demonstrated in the previous section that $50 \%$ AGA-UPy (AGA-UPy 50:50) sponges are softer, showing the greatest ability to create an adhesive matrix for NSCs. In this context, incorporation of UPy-dimers into the agarose backbone thus becomes a good method for enhancing tissue integration of neural biomaterials, with a great balance between mechanical and adhesive properties. 


\section{Conclusions}

The approaches discussed in this work improves upon the current sponge-like scaffold technology by providing a material with rapid cellular uptake triggered by the presence of cellular medium, growth factors, proteins, or nutrients. The supramolecular sponges have been shown to be very promising for biomedical applications because they delineate the mandatory use of cross-linking agents that often present toxicity. Moreover, these supramolecular sponges provide excellent physicochemical properties that render them useful scaffolds for minimally invasive neural implantation. These properties include: a micro-structure that is highly porous with inter-connected pores, shape preservation during gelation, the ability to recall water up to 100 times its weight, mechanical properties resembling those of human soft tissue, and finally, suitable cytocompatibility. Furthermore, UPy-modification was used to improve adhesion of neural stem cells encapsulated within the hydrogel and to guide neural adhesion at the interface with tissue, i.e., on the material surface. Thus, supramolecular sponges may be used as an innovative method for 3D scaffolds application and may also be utilized for many tissue engineering applications that include differentiation of cells in controlled microenvironments.

\section{Acknowledgment}

The authors thank FAPESP for financial support \#2017/11060-5 in the form of a postdoctoral grant to LCL and the thematic project \#2016/25406-5. We also thank the Brazilian Nanotechnology National Laboratory, LNNano, for access to microtomography analyses. The biological application of this work was supported by LABEX CORTEX (ANR-11-LABX-0042), LABEX DEVWECAN (ANR-10-LABX0061) of the University of Lyon within the program "Investissement d'Avenir" (ANR- 
11-IDEX-0007) operated by the French National Research Agency (ANR). We are also grateful for Adèle Fendler's precious help.

\section{Supporting Information}

The supporting information is available free of charge on the ACS Publications website: Experimental details: General procedure for the synthesis of Agarose-UPy. Figures: 1HNMR, Scheme of Agarose modification, FT-IR spectra, ${ }^{13} \mathrm{C}$ NMR, Stress-strain curve, SEM, Cell proliferation, and Confocal microscopy. Tables: Characteristic of FTIR bands and TGA analysis.

\section{Author Information \\ *Corresponding author: L. Lins, e-mail: luandaqme@gmail.com}

\section{References}

1. Lins, L. C.; Wianny, F.; Livi, S.; Hidalgo, I. A.; Dehay, C.; Duchet-Rumeau, J.; Gérard, J.-F., Development of Bioresorbable Hydrophilic-Hydrophobic Electrospun Scaffolds for Neural Tissue Engineering. Biomacromolecules 2016, 17, 3172-3187.

2. Lins, L. C.; Wianny, F.; Livi, S.; Dehay, C.; Duchet-Rumeau, J.; Gérard, J.-F., Effect of polyvinylidene fluoride electrospun fiber orientation on neural stem cell differentiation. J. Biomed. Mater. Res. 2017, 105, 2376-2393.

3. Erdem, A.; Ngwabebhoh, F. A.; Yildiz, U., Fabrication and characterization of soft macroporous Jeffamine cryogels as potential materials for tissue applications. RSC $A d v$. 2016, 6, 111872-111881.

4. Hixon, K. R.; Lu, T.; Sell, S. A., A comprehensive review of cryogels and their roles in tissue engineering applications. Acta Biomater. 2017, 62, 29-41.

5. $\quad$ Forget, A.; Christensen, J.; Lüdeke, S.; Kohler, E.; Tobias, S.; Matloubi, M.; Thomann, R.; Shastri, V. P., Polysaccharide hydrogels with tunable stiffness and provasculogenic properties via $\alpha$-helix to $\beta$-sheet switch in secondary structure. Proc. Natl. Acad. Sci. U. S. A. 2013, 110, 12887-12892.

6. Rao, S. S.; Winter, J. O., Adhesion molecule-modified biomaterials for neural tissue engineering. Front. Neuroeng. 2009, 2, 6-6. 
7. Lévesque, S. G.; Shoichet, M. S., Synthesis of cell-adhesive dextran hydrogels and macroporous scaffolds. Biomaterials 2006, 27, 5277-5285.

8. Huang, K.-F.; Hsu, W.-C.; Chiu, W.-T.; Wang, J.-Y., Functional improvement and neurogenesis after collagen-GAG matrix implantation into surgical brain trauma. Biomaterials 2012, 33, 2067-2075.

9. Bellamkonda, R.; Ranieri, J. P.; Aebischer, P., Laminin oligopeptide derivatized agarose gels allow three-dimensional neurite extension in vitro. J. Neurosci. Res. 1995, 41, 501-509.

10. Hirschberg, J. H. K. K.; Koevoets, R. A.; Sijbesma, R. P.; Meijer, E. W., Helical Supramolecular Aggregates Based on Ureidopyrimidinone Quadruple Hydrogen Bonding. Chem-Eur J 2003, 9, 4222-4231.

11. Dankers, P. Y. W.; Harmsen, M. C.; Brouwer, L. A.; Van Luyn, M. J. A.; Meijer, E. W., A modular and supramolecular approach to bioactive scaffolds for tissue engineering. Nat Mater 2005, 4, 568-574.

12. Dankers, P. Y. W.; van Leeuwen, E. N. M.; van Gemert, G. M. L.; Spiering, A. J. H.; Harmsen, M. C.; Brouwer, L. A.; Janssen, H. M.; Bosman, A. W.; van Luyn, M. J. A.; Meijer, E. W., Chemical and biological properties of supramolecular polymer systems based on oligocaprolactones. Biomaterials 2006, 27, 5490-5501.

13. Guan, Z.; Roland, J. T.; Bai, J. Z.; Ma, S. X.; McIntire, T. M.; Nguyen, M., Modular Domain Structure: A Biomimetic Strategy for Advanced Polymeric Materials. J. Am. Chem. Soc 2004, 126, 2058-2065.

14. Ren, W.; Li, Z.; Chen, Y.; Gao, H.; Yang, W.; Wang, Y.; Luo, Y., Facile Preparation of Linear Polyurethane from UPy-Capped Poly(dl-Lactic Acid) Macrodiol. Macromol. Mater. Eng. 2019, 304, 1800491.

15. Liu, S.; Qi, D.; Chen, Y.; Teng, L.; Jia, Y.; Ren, L., Quadruple hydrogen bonds and thermo-triggered hydrophobic interactions generate dynamic hydrogels to modulate transplanted cell retention. Biomater. Sci. 2019, 7, 1286-1298.

16. Folmer, B. J. B.; Sijbesma, R. P.; Versteegen, R. M.; van der Rijt, J. A. J.; Meijer, E. W., Supramolecular Polymer Materials: Chain Extension of Telechelic Polymers Using a Reactive Hydrogen-Bonding Synthon. Adv. Mater. 2000, 12, 874878.

17. Tamaru, S.-i.; Tokunaga, D.; Hori, K.; Matsuda, S.; Shinkai, S., Giant amino acids designed on the polysaccharide scaffold and their protein-like structural interconversion. Org. Biomol. Chem. 2014, 12, 815-822.

18. Wianny, F.; Bernat, A.; Huissoud, C.; Marcy, G.; Markossian, S.; Cortay, V.; Giroud, P.; Leviel, V.; Kennedy, H.; Savatier, P.; Dehay, C., Derivation and Cloning of a Novel Rhesus Embryonic Stem Cell Line Stably Expressing Tau-Green Fluorescent Protein. Stem Cells 2008, 26, 1444-1453.

19. Beijer, F. H.; Sijbesma, R. P.; Kooijman, H.; Spek, A. L.; Meijer, E. W., Strong Dimerization of Ureidopyrimidones via Quadruple Hydrogen Bonding. J. Am. Chem. Soc. 1998, 120, 6761-6769.

20. Keizer, Henk M.; Sijbesma, Rint P.; Meijer, E. W., The Convenient Synthesis of Hydrogen-Bonded Ureidopyrimidinones. Eur. J. Org. Chem. 2004, 2004, 2553-2555. 
21. Keizer, H. M.; van Kessel, R.; Sijbesma, R. P.; Meijer, E. W., Scale-up of the synthesis of ureidopyrimidinone functionalized telechelic poly(ethylenebutylene). Polymer 2003, 44, 5505-5511.

22. Jangizehi, A.; Ghaffarian, S. R.; Kowsari, E.; Nasseri, R., Supramolecular Polymer Based on Poly (Ethylene-co-Vinyl Alcohol)-g-Ureidopyrimidinone: SelfAssembly and Thermo-Reversibility. J. Macromol. Sci., Part B: Phys. 2014, 53, 848860 .

23. Kondaveeti, S.; Mehta, G. K.; Siddhanta, A. K., Modification of agarose: 6Aminoagarose mediated syntheses of fluorogenic pyridine carboxylic acid amides. Carbohydr. Polym. 2014, 106, 365-373.

24. Dankers, P. Y. W.; Adams, P. J. H. M.; Löwik, D. W. P. M.; van Hest, J. C. M.; Meijer, E. W., Convenient Solid-Phase Synthesis of Ureido-Pyrimidinone Modified Peptides. Eur. J. Org. Chem. 2007, 2007, 3622-3632.

25. Ren, W.; Li, Z.; Chen, Y.; Gao, H.; Yang, W.; Wang, Y.; Luo, Y., Facile Preparation of Linear Polyurethane from UPy-Capped Poly(dl-Lactic Acid) Macrodiol. Macromol. Mater. Eng. 2018, 1800491.

26. Wang, Y.; Zhang, X.; Han, N.; Wu, Y.; Wei, D., Oriented covalent immobilization of recombinant protein $\mathrm{A}$ on the glutaraldehyde activated agarose support. Int. J. Biol. Macromol. 2018, 120, 100-108.

27. Meena, R.; Siddhanta, A. K.; Prasad, K.; Ramavat, B. K.; Eswaran, K.; Thiruppathi, S.; Ganesan, M.; Mantri, V. A.; Rao, P. V. S., Preparation, characterization and benchmarking of agarose from Gracilaria dura of Indian waters. Carbohydr. Polym. 2007, 69, 179-188.

28. Zumbrink, T.; Demiroglou, A.; Jennissen, H. P., Analysis of affinity supports by 13C CP/MAS NMR spectroscopy: Application to carbonyldiimidazole- and novel tresyl chloride-synthesized agarose and silica gels. J. Mol. Recognit. 1995, 8, 363-373.

29. Hosseinpourpia, R.; Echart, A. S.; Adamopoulos, S.; Gabilondo, N.; Eceiza, A., Modification of Pea Starch and Dextrin Polymers with Isocyanate Functional Groups. Polymers 2018, 10, 939.

30. Despres, A.; Pizzi, A.; Delmotte, L., 13C NMR investigation of the reaction in water of UF resins with blocked emulsifiable isocyanates. J. Appl. Polym. Sci. 2006, 99, 589-596.

31. Manso Silván, M.; Messina, G. M. L.; Montero, I.; Satriano, C.; Ruiz, J. P. G.; Marletta, G., Aminofunctionalization and sub-micrometer patterning on silicon through silane doped agarose hydrogels. J. Mater. Chem. 2009, 19, 5226-5233.

32. Ravi, S.; Zhang, S.; Lee, Y.-R.; Kang, K.-K.; Kim, J.-M.; Ahn, J.-W.; Ahn, W.S., EDTA-functionalized KCC-1 and KIT-6 mesoporous silicas for $\mathrm{Nd} 3+$ ion recovery from aqueous solutions. $J$ Ind Eng Chem 2018, 67, 210-218.

33. Sánchez-Vaquero, V.; Satriano, C.; Tejera-Sánchez, N.; González Méndez, L.; García Ruiz, J. P.; Manso Silvána, M., Characterization and cytocompatibility of hybrid aminosilane-agarose hydrogel scaffolds. Biointerphases 2010, 5, 23-29.

34. Wu, S.; Yan, K.; Zhao, Y.; Tsai, C.-C.; Shen, J.; Bentley, W. E.; Chen, Y.; Deng, H.; Du, Y.; Payne, G. F.; Shi, X., Electrical Writing onto a Dynamically 
Responsive Polysaccharide Medium: Patterning Structure and Function into a Reconfigurable Medium. Adv. Funct. Mater. 2018, 28, 1803139.

35. Liu, Y.; Gaskell, K. J.; Cheng, Z.; Yu, L.; Payne, G. F., Chitosan-Coated Electrodes for Bimodal Sensing: Selective Post-Electrode Film Reaction for Spectroelectrochemical Analysis. Langmuir 2008, 24, 7223-7231.

36. Gauche, C.; Felisberti, M. I., Colloidal Behavior of Cellulose Nanocrystals Grafted with Poly(2-alkyl-2-oxazoline)s. ACS Omega 2019, 4, 11893-11905.

37. Lapprand, A.; Boisson, F.; Delolme, F.; Méchin, F.; Pascault, J. P., Reactivity of isocyanates with urethanes: Conditions for allophanate formation. Polym.

Degrad. Stab. 2005, 90, 363-373.

38. Nambu, Y.; Endo, T., Synthesis of novel aromatic isocyanurates by the fluoridecatalyzed selective trimerization of isocyanates. J Org Chem 1993, 58, 1932-1934.

39. Duong, H. A.; Cross, M. J.; Louie, J., N-Heterocyclic Carbenes as Highly Efficient Catalysts for the Cyclotrimerization of Isocyanates. Org. Lett. 2004, 6, 46794681.

40. Armstrong, G.; Buggy, M., Thermal stability of a ureidopyrimidinone model compound. Materials Science and Engineering: C 2001, 18, 45-49.

41. Andrienko, D., Introduction to liquid crystals. J. Mol. Liq. 2018, 267, 520-541.

42. Delgado, P. A.; Hillmyer, M. A., Combining block copolymers and hydrogen bonding for poly(lactide) toughening. RSC Adv. 2014, 4, 13266-13273.

43. Watase, M.; Nishinari, K.; Clark, A. H.; Ross-Murphy, S. B., Differential scanning calorimetry, rheology, x-ray, and NMR of very concentrated agarose gels. Macromolecules 1989, 22, 1196-1201.

44. Watase, M.; Nishinari, K., Rheology, DSC and Volume or Weight Change Induced by Immersion in Solvents for Agarose and Kappa-Carrageenan Gels. Polym. J. 1986, 18, 1017.

45. Kittur, F. S.; Harish Prashanth, K. V.; Udaya Sankar, K.; Tharanathan, R. N., Characterization of chitin, chitosan and their carboxymethyl derivatives by differential scanning calorimetry. Carbohydr. Polym. 2002, 49, 185-193.

46. Demarger-Andre, S.; Domard, A., Chitosan carboxylic acid salts in solution and in the solid state. Carbohydr. Polym. 1994, 23, 211-219.

47. Braccini, I.; Rodríguez-Carvajal, M. A.; Pérez, S., Chain-Chain Interactions for Methyl Polygalacturonate: Models for High Methyl-Esterified Pectin Junction Zones. Biomacromolecules 2005, 6, 1322-1328.

48. $\quad$ Chung, T. W.; Yang, J.; Akaike, T.; Cho, K. Y.; Nah, J. W.; Kim, S. I.; Cho, C. S., Preparation of alginate/galactosylated chitosan scaffold for hepatocyte attachment. Biomaterials 2002, 23, 2827-2834.

49. Charlot, A.; Auzély-Velty, R.; Rinaudo, M., Specific Interactions in Model Charged Polysaccharide Systems. J. Phys. Chem. B 2003, 107, 8248-8254.

50. Nadgorny, M.; Collins, J.; Xiao, Z.; Scales, P. J.; Connal, L. A., 3D-printing of dynamic self-healing cryogels with tuneable properties. Polym. Chem. 2018, 9, 16841692. 
51. Cheng, J.; Amin, D.; Latona, J.; Heber-Katz, E.; Messersmith, P. B., Supramolecular Polymer Hydrogels for Drug-Induced Tissue Regeneration. ACS Nano 2019, 13, 5493-5501.

52. Mihajlovic, M.; Staropoli, M.; Appavou, M.-S.; Wyss, H. M.; PyckhoutHintzen, W.; Sijbesma, R. P., Tough Supramolecular Hydrogel Based on Strong Hydrophobic Interactions in a Multiblock Segmented Copolymer. Macromolecules 2017, 50, 3333-3346.

53. Seiffert, S.; Sprakel, J., Physical chemistry of supramolecular polymer networks. Chem. Soc. Rev. 2012, 41, 909-930.

54. Li, J.; Sullivan, K. D.; Brown, E. B.; Anthamatten, M., Thermally activated diffusion in reversibly associating polymers. Soft Matter 2010, 6, 235-238.

55. Knowlton, E. D.; Pine, D. J.; Cipelletti, L., A microscopic view of the yielding transition in concentrated emulsions. Soft Matter 2014, 10, 6931-6940.

56. Glassman, M. J.; Chan, J.; Olsen, B. D., Reinforcement of Shear Thinning Protein Hydrogels by Responsive Block Copolymer Self-Assembly. Adv. Funct. Mater. 2013, 23, 1182-1193.

57. Loontjens, T.; Put, J.; Coussens, B.; Lange, R.; Palmen, J.; Sleijpen, T.; Plum, B., Novel supramolecular polymer networks based on melamine- and imide-containing oligomers. Macromol. Symp. 2001, 174, 357-371.

58. Buhler, E.; Candau, S.-J.; Kolomiets, E.; Lehn, J.-M., Dynamical properties of semidilute solutions of hydrogen-bonded supramolecular polymers. Phys. Rev. E 2007, 76, 061804.

59. Beaucage, G., Small-Angle Scattering from Polymeric Mass Fractals of Arbitrary Mass-Fractal Dimension. J. Appl. Crystallogr. 1996, 29, 134-146.

60. Bin Imran, A.; Esaki, K.; Gotoh, H.; Seki, T.; Ito, K.; Sakai, Y.; Takeoka, Y., Extremely stretchable thermosensitive hydrogels by introducing slide-ring polyrotaxane cross-linkers and ionic groups into the polymer network. Nat. Commun. 2014, 5, 5124.

61. Shibayama, M., Small-angle neutron scattering on polymer gels: phase behavior, inhomogeneities and deformation mechanisms. Polymer Journal 2011, 43, 18-34.

62. Santinath Singh, S.; Aswal, V. K.; Bohidar, H. B., Internal structures of agargelatin co-hydrogels by light scattering, small-angle neutron scattering and rheology. Eur. Phys. J. E 2011, 34, 62.

63. Newbloom, G. M.; Kim, F. S.; Jenekhe, S. A.; Pozzo, D. C., Mesoscale Morphology and Charge Transport in Colloidal Networks of Poly(3-hexylthiophene). Macromolecules 2011, 44, 3801-3809.

64. Zhao, S.; Malfait, W. J.; Guerrero-Alburquerque, N.; Koebel, M. M.; Nyström, G., Biopolymer Aerogels and Foams: Chemistry, Properties, and Applications. Angew. Chem., Int. Ed. 2018, 57, 7580-7608.

65. Martoïa, F.; Cochereau, T.; Dumont, P. J. J.; Orgéas, L.; Terrien, M.; Belgacem, M. N., Cellulose nanofibril foams: Links between ice-templating conditions, microstructures and mechanical properties. Mater. Des. 2016, 104, 376-391.

66. Delcroix, G. J. R.; Schiller, P. C.; Benoit, J.-P.; Montero-Menei, C. N., Adult cell therapy for brain neuronal damages and the role of tissue engineering. Biomaterials 2010, 31, 2105-2120. 
67. Arregui, C. O.; Carbonetto, S.; McKerracher, L., Characterization of neural cell adhesion sites: point contacts are the sites of interaction between integrins and the cytoskeleton in PC12 cells. J. Neurosci. 1994, 14, 6967-6977.

68. Hynes, R. O., Integrins: Versatility, modulation, and signaling in cell adhesion. Cell 1992, 69, 11-25.

69. Hall, P. E.; Lathia, J. D.; Miller, N. G.; Caldwell, M. A.; ffrench-Constant, C., Integrins are markers of human neural stem cells. Stem Cells 2006, 24, (9), 2078-84.

70. Clark, E. A.; Brugge, J. S., Integrins and signal transduction pathways: the road taken. Science 1995, 268, 233-239.

71. Kuo, J.-C., Chapter Three - Focal Adhesions Function as a Mechanosensor. In Progress in Molecular Biology and Translational Science, Engler, A. J.; Kumar, S., $1^{\text {st }}$ Eds. Academic Press. 2014, 126, 55-73.

72. Akiyama, S. K., Integrins in cell adhesion and signaling. Human cell 1996, 9, 181-186.

73. Jembrek, M. J.; Šimić, G.; Hof, P. R.; Šegota, S., Atomic force microscopy as an advanced tool in neuroscience. Transl. Neurosci. 2015, 6, 117-130.

74. Chen, C. S.; Mrksich, M.; Huang, S.; Whitesides, G. M.; Ingber, D. E., Geometric Control of Cell Life and Death. Science 1997, 276, 1425-1428.

75. Loubet, D.; Dakowski, C.; Pietri, M.; Pradines, E.; Bernard, S.; Callebert, J.; Ardila-Osorio, H.; Mouillet-Richard, S.; Launay, J.-M.; Kellermann, O.; Schneider, B., Neuritogenesis: the prion protein controls $\beta 1$ integrin signaling activity. FASEB $J \mathbf{2 0 1 2}$, 26, 678-690.

76. Zustiak, S. P.; Wei, Y.; Leach, J. B., Protein-hydrogel interactions in tissue engineering: mechanisms and applications. Tissue Eng. Part B Rev. 2013, 19, 160-71.

77. Lee, M. H.; Brass, D. A.; Morris, R.; Composto, R. J.; Ducheyne, P., The effect of non-specific interactions on cellular adhesion using model surfaces. Biomaterials 2005, 26, 1721-1730.

78. Faucheux, N.; Schweiss, R.; Lützow, K.; Werner, C.; Groth, T., Self-assembled monolayers with different terminating groups as model substrates for cell adhesion studies. Biomaterials 2004, 25, 2721-2730.

79. Roach, P.; Parker, T.; Gadegaard, N.; Alexander, M. R., Surface strategies for control of neuronal cell adhesion: A review. Surf. Sci. Rep. 2010, 65, 145-173.

80. Bacáková, L.; Filová, E.; Rypácek, F.; Svorcík, V.; Starý, V., Cell adhesion on artificial materials for tissue engineering. Physiol Res 2004, 53, 35-45.

81. Alberts, B.; Johnson, A.; Lewis, J.; Raff, M.; Roberts, K.; Walter, P., Cell Junctions, Cell Adhesion, and the Extracellular Matrix. Mol. Biol. Cell 2002, Chapter 19,4 th ed, 1, $212-214$.

82. Gentile, F.; Tirinato, L.; Battista, E.; Causa, F.; Liberale, C.; di Fabrizio, E. M.; Decuzzi, P., Cells preferentially grow on rough substrates. Biomaterials 2010, 31, 72057212.

83. Khan, S. P.; Auner, G. G.; Newaz, G. M., Influence of nanoscale surface roughness on neural cell attachment on silicon. Nanomed-Nanotechnol 2005, 1, 125129. 
84. Decuzzi, P.; Ferrari, M., The adhesive strength of non-spherical particles mediated by specific interactions. Biomaterials 2006, 27, 5307-5314.

85. Georges, P. C.; Miller, W. J.; Meaney, D. F.; Sawyer, E. S.; Janmey, P. A., Matrices with Compliance Comparable to that of Brain Tissue Select Neuronal over Glial Growth in Mixed Cortical Cultures. Biophys. J. 2006, 90, 3012-3018. 


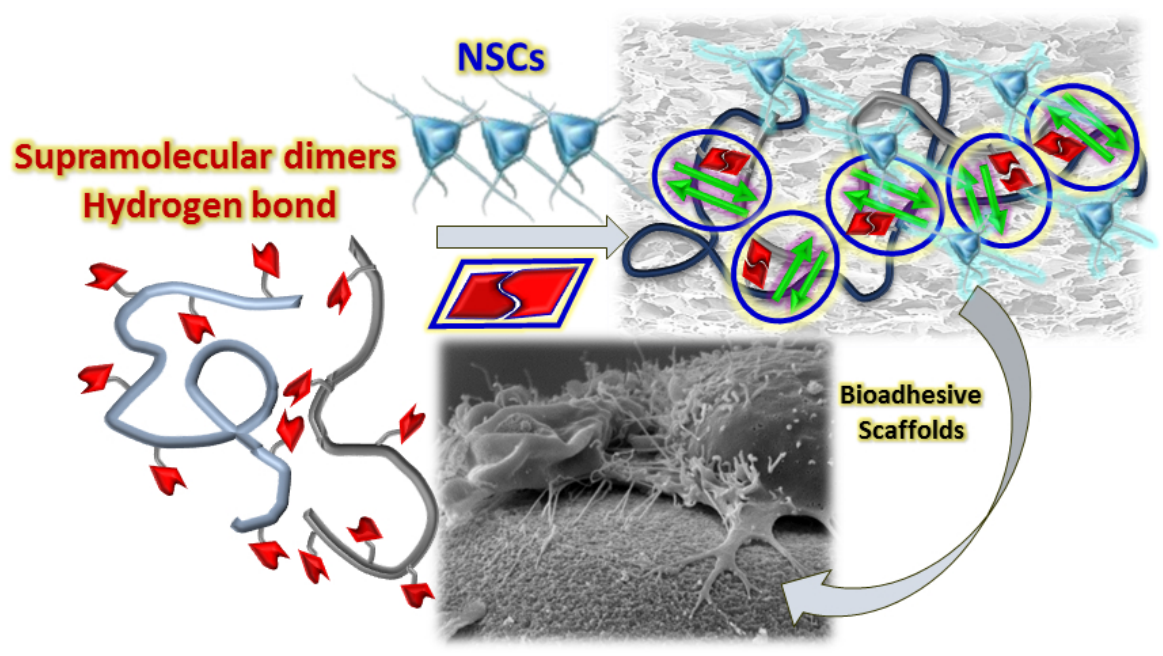

$254 \times 190 \mathrm{~mm}(96 \times 96 \mathrm{DPI})$ 